,pdfcreator $=$ HAL,pdfproducer $=$ PDFLaTeX,pdfsubject $=$ Mathematics $[$ math $] /$ Complex Variables [math.CV]

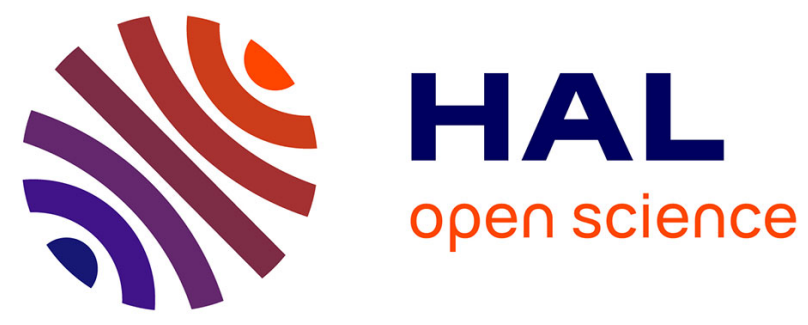

\title{
Estimates for some Weighted Bergman Projections
}

Philippe Charpentier, Yves Dupain, Modi Mounkaila,

\section{To cite this version:}

Philippe Charpentier, Yves Dupain, Modi Mounkaila,. Estimates for some Weighted Bergman Projections. Complex Variables and Elliptic Equations, 2014, 59 (8), pp.1070-1095. 10.1080/17476933.2013.805411. hal-00761375v3

\section{HAL Id: hal-00761375 \\ https://hal.science/hal-00761375v3}

Submitted on 23 May 2013

HAL is a multi-disciplinary open access archive for the deposit and dissemination of scientific research documents, whether they are published or not. The documents may come from teaching and research institutions in France or abroad, or from public or private research centers.
L'archive ouverte pluridisciplinaire HAL, est destinée au dépôt et à la diffusion de documents scientifiques de niveau recherche, publiés ou non, émanant des établissements d'enseignement et de recherche français ou étrangers, des laboratoires publics ou privés. 


\title{
ESTIMATES FOR WEIGHTED BERGMAN PROJECTIONS ON PSEUDO-CONVEX DOMAINS OF FINITE TYPE IN $\mathbb{C}^{n}$
}

\author{
P. CHARPENTIER, Y. DUPAIN \& M. MOUNKAILA
}

\begin{abstract}
In this paper we investigate the regularity properties of weighted Bergman projections for smoothly bounded pseudo-convex domains of finite type in $\mathbb{C}^{n}$. The main result is obtained for weights equal to a non-negative rational power of the absolute value of a special defining function $\rho$ of the domain: we prove (weighted) Sobolev- $L^{p}$ and Lipschitz estimates for domains in $\mathbb{C}^{2}$ (or, more generally, for domains having a Levi form of rank $\geq n-2$ and for "decoupled" domains) and for convex domains. In particular, for these defining functions, we generalize results obtained by A. Bonami \& S. Grellier and D. C. Chang \& B. Q. Li. We also obtain a general (weighted) Sobolev- $L^{2}$ estimate.
\end{abstract}

\section{INTRODUCTION}

Let $\Omega$ be a bounded open set in $\mathbb{C}^{n}$. Let $\omega$ be a non-negative measurable function on $\Omega$ and $\lambda$ be the Lebesgue measure on $\mathbb{C}^{n}$. The function $\omega$ is called an admissible weight (or simply a weight) for $\Omega$ if the set $A^{2}(\Omega, \omega d \lambda)$ of square integrable holomorphic functions with respect to the measure $\omega d \lambda$ is a closed subspace of the Hilbert space $L^{2}(\Omega, \omega d \lambda)$ (see [PW90]). So, if $\omega$ is a weight on $\Omega$, the weighted Bergman projection $P_{\omega}^{\Omega}$, i.e. the orthogonal projection of $L^{2}(\Omega, \omega d \lambda)$ onto $A^{2}(\Omega, \omega d \lambda)$, is well-defined.

The aim of this paper is to investigate Lipschitz and Sobolev $L^{p}$ regularities of $P_{\omega}^{\Omega}$ when $\Omega$ is smooth, pseudo-convex and of finite type.

As far as we know, for general finite type domains, only two results were previously known. First, in [BG95], A. Bonami \& S. Grellier proved Lipschitz and Sobolev $L^{p}$ estimates for the weighted Bergman projection $P_{\omega}^{\Omega}$ of a finite type domain in $\mathbb{C}^{2}$ when the weight $\omega$ is a non-negative entire power of the absolute value of a defining function $\rho$ of the domain (i.e. $\omega=(-\rho)^{q}$, $q \in \mathbb{N}$ ). Secondly, in [CL97], D. C. Chang \& B. Q. Li extended these results to "decoupled" domains in $\mathbb{C}^{n}$.

The main results of the present paper extend, for special defining functions $\rho$, those estimates of $P_{\omega}^{\Omega}$ in two directions. First we extend the class of weights $\omega$ to non-negative rational powers of $|\rho|$ (i.e. $\omega=(-\rho)^{r}, r \in \mathbb{Q}$ ). Second we extend the class of domains to include convex domains (of finite type).

Moreover, we obtain weighted $L^{2}$-Sobolev regularity of $P_{(-\rho)^{r}}^{\Omega}$ for general pseudo-convex domains of finite type in $\mathbb{C}^{n}, \rho$ being also a special defining function of $\Omega$.

In complex analysis the (weighted or not) Bergman projection plays a fundamental role and its regularity has been extensively studied.

A fundamental class of weights is the one introduced by L. Hörmander in [Hör65] in order to solve the $\bar{\partial}$-equation. Let $\varphi$ be a pluri-subharmonic function defined in $\Omega$. Hörmander's theorem solves the so-called $\bar{\partial}_{\varphi}$-Neumann problem associated to the weight $e^{-\varphi}$ proving the existence of the Neumann operator $\mathscr{N}_{\varphi}$ inverting the complex laplacian $\square_{\varphi}$. Recall that the Bergman projection $P_{e^{-\varphi}}^{\Omega}$ is closely related to $\mathscr{N}_{\varphi}$ by the formula $P_{e^{-\varphi}}^{\Omega}=\mathrm{Id}-\bar{\partial}_{\varphi}^{*} \mathscr{N}_{\varphi} \bar{\partial}_{\varphi}$.

For $\varphi=0$ many results have been obtained in this direction in various function spaces. In particular, for $\left(L^{2}\right)$ Sobolev regularity there is a very large bibliography essentially based on J. J. Kohn's work (see [Str10] for a good general presentation). For other spaces, a lot of sharp results were obtained by several authors, but there are still basic open problems (see for example [NRSW89, CNS92, BC00, McN94, MS94, Cho96, MS97, Cho03, CD06b, CD06a, CD08] and references therein).

For non-zero functions $\varphi$, the only general result, due to J. J. Kohn ([Koh73]), gives $L^{2}$-Sobolev estimates for the $\bar{\partial}_{\varphi}$-Neumann problem for general smoothly bounded pseudo-convex domains with $\varphi=t|z|^{2}$, where $t$ is big enough depending on the Sobolev scale. Recall that there exist smoothly bounded pseudo-convex domains for which the (unweighted) Bergman projection is not $L^{2}$-Sobolev regular ([Chr96]). However, if $\Omega$ is of finite type, it is not difficult to see that, if $\varphi$ is $\mathscr{C}^{\infty}$ on $\bar{\Omega}$ then the weighted Bergman projection $P_{e^{-}}^{\Omega}$ has the same $L^{2}$-Sobolev regularity than the unweighted one.

For the Bergman projection $P_{\omega}^{\Omega}$ with a general (admissible) weight $\omega$, very few results were obtained for finite type domains. In addition to the results of A. Bonami \& S. Grellier and D. C. Chang \& B. Q. Li cited before, sharp results were obtained for strictly pseudo-convex domains. In the case of the unit ball of $\mathbb{C}^{n}$, for weights equal to a power greater than -1 of $1-|z|^{2}$, the kernels of these operators can be written explicitly (see [Cha80, HP84b, HP84a]) and then it is possible to obtain very precise estimates. Generalizations of these results to strictly pseudo-convex domains have also been done by several authors (see [LR86, LR87, LR88, Cum90]).

2000 Mathematics Subject Classification. 32F17, 32T25, 32T40.

Key words and phrases. pseudo-convex, finite type, Levi form locally diagonalizable, convex, extremal basis, geometric separation, weighted Bergman projection, $\bar{\partial}_{\varphi}$-Neumann problem. 
Even in dimension 1, $L^{p}$ estimates for weighted Bergman projections can be true only for $p=2$, and, in general, are not easy to obtain, as shown in [Zey11b, Zey11a, Zeya, Zeyb].

The method used in this paper is completely different than that used in A. Bonami \& S. Grellier or D. C. Chang \& B. Q. Li papers. It is inspired by a well-known method introduced by F. Forelli and W. Rudin (see [FR75, Rud80, Lig89]): we look at $\Omega$ as a slice of a pseudo-convex domain $\widetilde{\Omega}$ of finite type in $\mathbb{C}^{n+m}$ and try to deduce estimates for weighted Bergman projections of $\Omega$ from estimates of the unweighted Bergman projection of $\widetilde{\Omega}$.

The paper is organized as follows. In the first section we present the main results on weighted Bergman projections. In Section 2 we define the domain $\widetilde{\Omega}$ and discuss its fundamental properties. In Section 3 we give the basic relations between the Bergman projection of $\widetilde{\Omega}$ and a weighted Bergman projection of $\Omega$ and prove the general $L^{2}$-Sobolev results. In Section 4, we prove the $L_{s}^{p}$ and Lipschitz estimates given in Theorem 1.1 establishing sharp estimates of the kernels of weighted Bergman projections.

\section{MAIN RESUlTS AND METHODS}

For simplicity, we only state here the main result concerning the Bergman projection for weights equal to a non-negative rational power of $|\rho|$ where $\rho$ is a particular defining function of $\Omega$. Detailed results for other operators, other weights and for the Bergman kernel will be given in the next sections.

If $k$ is a positive function on an open set $\Omega$ in $\mathbb{C}^{n}$ whose inverse is locally integrable, we denote by $P_{k}=P_{k}^{\Omega}$ the orthogonal projection of the Hilbert space $L^{2}(k d \lambda)$ onto the (closed [PW90]) subspace of holomorphic functions (i.e. the Bergman projection associated to the weight $k$ ).

Theorem 1.1. Let $\Omega$ be a smoothly bounded pseudo-convex domain of finite type $M$ and $\rho$ a defining function of $\Omega$. We assume that one of the two following conditions is satisfied:

- $\Omega$ is a domain in $\mathbb{C}^{2}$ and $\rho$ is such that there exists $\left.\left.s \in\right] 0,1\right]$ such that $-(-\rho)^{s}$ is strictly pluri-subharmonic in $\Omega$ ([DF77a]);

- $\Omega$ is convex and, if $g$ is a gauge of $\Omega$, then $\rho=g^{4} e^{1-1 / g}-1$.

Let $\omega=(-\rho)^{r}$ with $r$ a non-negative rational number. Let us denote by $P_{\omega}^{\Omega}$ the weighted Bergman projection of $\Omega$ associated to the Hilbert space $L^{2}(\omega d \lambda)$, d $\lambda$ denoting the Lebesgue measure. Let us also denote by $\delta_{\partial \Omega}$ the distance to the boundary of $\Omega$.

(1) Let $s \in \mathbb{N}$. Then, for $p \in] 1,+\infty\left[\right.$ and $-1<\beta<p(r+1)-1, P_{\omega}^{\Omega}$ maps the Sobolev space $L_{s}^{p}\left(\delta_{\partial \Omega}^{\beta}\right)$ continuously into itself.

(2) For $\alpha<1 / M, P_{\omega}^{\Omega}$ maps the Lipschitz space $\Lambda_{\alpha}$ continuously into itself.

For $L^{2}$-Sobolev estimates, we have more general results. For example, for weighted Bergman projections:

Theorem 1.2. Let $\Omega$ be any smoothly bounded pseudo-convex domain of finite type in $\mathbb{C}^{n}$. Let $\rho$ be a smooth defining function of $\Omega$ such that there exists $s \in] 0,1]$ such that $-(-\rho)^{s}$ is strictly pluri-subharmonic in $\Omega$. Let $r \in \mathbb{Q}+$. Let $\omega=(-\rho)^{r}$. Then the weighted Bergman projection $P_{\omega}^{\Omega}$ associated to the Hilbert space $L^{2}(\omega d \lambda)$ maps the Sobolev space $L_{s}^{2}(\omega d \lambda)$ continuously into itself for all $s \in \mathbb{N}$.

The method we use is a modification of a well-known construction of Forelli-Rudin. Such method has been used by several authors for the same kind of studies (for example E. Ligocka and Y. Zeytuncu [Lig89, Zeyb]). To try to obtain weights which are not only integer powers of the absolute value of the defining function of $\Omega$, we consider a more general situation investigating the properties of a domain $\widetilde{\Omega}$ defined in $\mathbb{C}^{n+m}$ by an equation of the form $\rho(z)+h(w)<0$ where $\rho$ is a defining function of $\Omega$ and $h$ a positive function. These properties are discussed in Section 2.

Remark.

(1) The restriction $r \in \mathbb{Q}_{*}^{+}$in our results is due to the method and we do not know if the theorems can be extended to the natural scale of powers which is $]-1,+\infty[$.

(2) Recall that the results of A. Bonami \& S. Grellier and D. C. Chang \& B. Q. Li are valid for any defining function of $\Omega$. It seems that it is not easy to extend our results to any defining function. We will return to this question in a future paper.

The only thing we can say in general (i.e. without the finite type hypothesis) is that the set of weights for which the corresponding weighted Bergman projections satisfy a given estimate is, in a certain sense, open. This can be seen using a special case of [PW90, Theorem 4.2] (which can be easily directly proved): let $\omega$ be an admissible weight, $h$ be a real smooth function such that $0<c \leq|h| \leq C<1$, and denotes $M_{h}$ the operator $g \mapsto h g$. Then

$$
P_{(1+h) \omega}^{\Omega}=P_{\omega}^{\Omega}+\sum_{k=1}^{\infty}(-1)^{k-1}\left[P_{\omega}^{\Omega} \circ M_{h}\right]^{k} \circ\left(\mathrm{Id}-P_{\omega}^{\Omega}\right)
$$

where $[\ldots]^{k}$ is the power for the composition operator, the series converging in the operator norm of $\mathscr{L}\left(L^{2}(\omega d \lambda)\right)$. For example, if $P_{\omega}^{\Omega}$ maps $L^{p}\left(\delta_{\partial \Omega}^{\beta}\right)$ continuously into itself with norm $K_{p, \beta}^{\omega}$, then, a simple calculation shows immediately that the same is true for $P_{(1+h) \omega}^{\Omega}$ if $\|h\|_{\infty} \leq \frac{1}{\left(K_{p, \beta}^{\omega}+1\right)^{2}}$. 


\section{A HaRtogs domain $\widetilde{\Omega}$ IN $\mathbb{C}^{n+m}$ BASED ON A DOMAIN $\Omega$ IN $\mathbb{C}^{n}$}

For a given smoothly bounded pseudo-convex domain $\Omega$ in $\mathbb{C}^{n}$ with a defining function $\rho$, we consider a smooth non-negative function $h$ defined in $\mathbb{C}^{m}$ such that

$$
h(w)=0 \Leftrightarrow w=0 \text { and } \lim _{|w| \rightarrow+\infty} h(w)=+\infty
$$

and we denote by $\widetilde{\Omega}$ the smooth bounded domain

$$
\widetilde{\Omega}=\left\{(z, w) \in \mathbb{C}^{n} \times \mathbb{C}^{m} \text {, s. t. } r(z, w)=\rho(z)+h(w)<0\right\} .
$$

Then, for particular functions $h$, there are very simple relations between the standard Bergman kernel of $\widetilde{\Omega}$ and a weighted Bergman kernel of $\Omega$, and between the unweighted $\bar{\partial}$-Neumann problem on $\widetilde{\Omega}$ and a weighted $\bar{\partial}_{\varphi}$-Neumann problem on $\Omega$ (see Section 3). The goal of this part is to find what conditions on $\rho$ and $h$ would provide enough properties on $\widetilde{\Omega}$ so that we can obtain sharp estimates on the Bergman projection (or on the $\bar{\partial}$-Neumann problem of $\widetilde{\Omega}$ ) or sufficiently precise information on the Bergman kernel of $\widetilde{\Omega}$ on $\{w=0\}$.

More precisely, in this section we discuss the following questions: suppose $\nabla h(w) \neq 0$ if $w \neq 0$; under what conditions on $\rho$ and $h$ the domain $\widetilde{\Omega}$ is:

- pseudo-convex;

- pseudo-convex of finite type if $\Omega$ is of finite type.

- completely geometrically separated, in the sense of [CD08] (at every boundary point or at boundary points near $\{w=0\}$ ) if $\Omega$ is so.

To get these properties, quite strong conditions have to be imposed to $\rho$ and $h$. To simplify the reading of the paper, we first state these different conditions.

\subsection{A special defining function for $\Omega$}

As we will see in Section 2.3, even with the function $h(w)=|w|^{2}, w \in \mathbb{C}$, and for very simple domains like the unit ball, $\widetilde{\Omega}$ is not automatically pseudo-convex: this depends on the choice of the defining function $\rho$ (Remark 2.2).

Fortunately, a good choice can always be done using a celebrated theorem of K. Diederich \& J. E. Fornæss ([DF77a, Theorem 1]) which proves that for any smooth bounded pseudo-convex domain $\Omega$ there exists $\left.\left.s_{\Omega} \in\right] 0,1\right]$ such that, for $\left.s \in\right] 0, s_{\Omega}[$ there exists a smooth defining function $\rho$ of $\Omega$ such that the function $-(-\rho)^{s}$ is strictly pluri-subharmonic in $\Omega$. Then, to fix the notations:

Throughout this paper, for $s \in] 0, s_{\Omega}\left[\right.$, we will denote by $\rho_{s}$ a defining function such that

$$
-\left(-\rho_{S}\right)^{s} \text { is strictly pluri-subharmonic in } \Omega \text {. }
$$

Of course such a function $\rho_{s}$ is not unique.

Remark 2.1.

(1) The pluri-subharmonicity of $-\left(-\rho_{s}\right)^{s}$ means

$$
i \partial \bar{\partial} \rho_{s} \geq i \frac{1-s}{\rho_{s}} \partial \rho_{s} \wedge \bar{\partial} \rho_{s} .
$$

Thus $i \partial \bar{\partial} \rho_{s} \geq \frac{i}{\rho_{s}} \partial \rho_{s} \wedge \bar{\partial} \rho_{s}$, and, as this means that $-\log \left(-\rho_{s}\right)$ is pluri-subharmonic in $\Omega$, and the $\bar{\partial}_{-r \log \left(-\rho_{s}\right)}$-Neumann problem is well-defined for $r \geq 0$.

(2) Let $U$ be an open set in $\mathbb{C}^{n}$. Let $\rho$ be a $\mathscr{C}^{\infty}$ function on $U$ whose gradient does not vanish. Assume there exists $z^{0} \in U$ such that $\rho\left(z^{0}\right)=0$. Thus, for $\varepsilon \in \mathbb{R}$ sufficiently small the set

$$
\Xi=\{z \in U \text { such that } \rho(z)=\varepsilon\}
$$

is a non-empty smooth hypersurface. Assume moreover that $i \partial \bar{\partial} \rho \geq i \frac{\mu}{\rho} \partial \rho \wedge \bar{\partial} \rho, \mu \in \mathbb{R}$, on $U$. Then, at every point of $\Xi$, the restriction of $i \partial \bar{\partial} \rho$ to the complex tangent space of $\Xi$ is non-negative.

\subsection{Hypothesis on the function $h$}

Depending on the properties we want to have for $\widetilde{\Omega}$, several conditions will be imposed on $h$. We define now five conditions that we will use in the following sections.

Let $\Omega$ be a bounded smooth pseudo-convex domain in $\mathbb{C}^{n}$ and let $h$ be a smooth real function on $\mathbb{C}^{m}$.

Condition I:

$\rho=\rho_{s}$ is a defining function of $\Omega$ satisfying (2.2) (Section 2.1). $h$ is non-negative, $h(w)=0 \Leftrightarrow w=0$ (and thus $\nabla h(0)=0), \nabla h(w) \neq 0$ if $w \neq 0, \lim _{|w| \rightarrow+\infty} h(w)=+\infty$ and there exists $s^{\prime} \in\left[0, s\left[\right.\right.$ such that $h^{s^{\prime}}$ is pluri-subharmonic (i.e. $i \partial \bar{\partial} h \geq i \frac{1-s^{\prime}}{h} \partial h \wedge \bar{\partial} h$ which implies that $h$ is strictly pluri-subharmonic at every point $w$ such that $\frac{\partial h}{\partial w_{i}}(w) \neq 0$ for all $\left.i\right)$.

\section{Condition II:}

$h(w)=\sum_{i=1}^{p} h_{i}\left(w_{i}\right), w=\left(w_{1}, \ldots, w_{p}\right), w_{i} \in \mathbb{C}^{m_{i}}$, the functions $h_{i}$ being non-negative smooth pluri-subharmonic on $\mathbb{C}^{m_{i}}$, and satisfying: 
(a) for every $i, h_{i}\left(w_{i}\right)=0 \Leftrightarrow w_{i}=0, \nabla h_{i}\left(w_{i}\right) \neq 0$ if $w_{i} \neq 0, \lim _{|w| \rightarrow+\infty} h_{i}(w)=+\infty$;

(b) $\log \left(h_{i}\right)$ is pluri-subharmonic (i.e. $i \partial \bar{\partial} h_{i} \geq i \frac{1}{h_{i}} \partial h_{i} \wedge \bar{\partial} h_{i}$ ) and $h_{i}$ is strictly pluri-subharmonic outside the origin;

(c) $h_{i}$ is of finite type $2 q_{i}=\operatorname{typ}_{0}\left(h_{i}\right)$ at the origin (in the sense introduced at the beginning of Section 2.4).

\section{Condition III:}

$h$ satisfies Condition II with $m_{i}=1$ for every $i$ (thus the first part of the second condition of Condition II means $\triangle h_{i} \geq$ $\left.\frac{1}{h_{i}}\left|h_{i}^{\prime}\right|^{2}\right)$, and, for each $i$ there exists a function $\alpha_{i}, \mathscr{C}^{\infty}$ in a neighborhood of the origin in $\mathbb{C}, \lim _{w_{i} \rightarrow 0} \alpha_{i}\left(w_{i}\right)=0$, such that $\frac{\partial h_{i}}{\partial w_{i}}=\alpha_{i} \frac{\partial^{2} h_{i}}{\partial w_{i} \partial \bar{w}_{i}}$ in that neighborhood.

\section{Condition IV:}

$\Omega$ is of finite type, $h$ satisfies Condition III, and, for each $i, h_{i}\left(w_{i}\right)=k_{i}\left(\left|w_{i}\right|\right), k_{i}(t) \asymp t^{2 q_{i}}$ (where $f \asymp g$ means that there exist two constants $c>0$ and $C>0$ such that $c f \leq g \leq C f)$ and $2 q_{i}$ strictly larger than the type of $\Omega$.

\section{Condition V:}

$\Omega$ is of finite type, $h$ satisfies Condition II with $m_{i}=1$ for every $i$, and, for each $i,\left|\frac{\partial^{2} h_{i}}{\partial w_{i}^{2}}\right| \leq \triangle h_{i}, h_{i}\left(w_{i}\right)=k_{i}\left(\left|w_{i}\right|\right)$ and $k_{i}(t) \asymp t^{2 q_{i}}$ and $q_{i}$ strictly larger than the type of $\Omega$.

Condition I is used in Section 2.3 to get the pseudo-convexity of $\widetilde{\Omega}$. Condition II is used in Section 2.4 to ensure that $\widetilde{\Omega}$ is of finite type. In Section 2.5 we use Condition III to obtain that $\widetilde{\Omega}$ have a Levi form locally diagonalizable (and thus is "completely geometrically separated" ([CD08])) when $\Omega$ is a finite type domain in $\mathbb{C}^{2}$. Finally, Conditions IV and V are used in Sections 4.1 and 4.2 to get pointwise estimates of a weighted Bergman kernel in these two cases.

Example 2.1. Let $m_{i} \in \mathbb{N}_{*}, 1 \leq i \leq p, m=\sum_{i} m_{i}, q_{i} \in \mathbb{N}_{*}, 1 \leq i \leq p$. Then the function

$$
h: w=\left(w_{1}, \ldots w_{p}\right) \in \prod_{i} \mathbb{C}^{m_{i}}=\mathbb{C}^{m} \mapsto \sum_{i}\left|w_{i}\right|^{2 q_{i}}
$$

satisfies Conditions I (whatever $s$ ) and II, and all other conditions if $m_{i}=1$ for every $i$ and the $q_{i}$ are large enough.

Proof. Let us denote $h_{i}\left(w_{i}\right)=\left|w_{i}\right|^{2 q_{i}}, 1 \leq i \leq p$. Then $\bar{\partial} h=\sum_{i} \bar{\partial} h_{i}, \partial \bar{\partial} h=\sum_{i} \partial \bar{\partial} h_{i}$ and, a simple calculation and Cauchy-Schwarz inequality give, for $1 \leq i \leq p$,

$$
\begin{aligned}
i \partial \bar{\partial} h_{i}= & i q_{i}^{2}\left|w_{i}\right|^{2 q_{i}-4}\left(\sum_{j} \overline{w_{i}^{j}} d w_{i}^{j}\right) \wedge\left(\sum_{j} w_{i}^{j} \overline{d w_{i}^{j}}\right)+ \\
& +i q_{i}\left|w_{i}\right|^{2 q_{i}-2}\left(\sum_{j} d w_{i}^{j} \wedge \overline{d w_{i}^{j}}\right)-i q_{i}\left|w_{i}\right|^{2 q_{i}-4}\left(\sum_{j} \overline{w_{i}^{j}} d w_{i}^{j}\right) \wedge\left(\sum_{j} w_{i}^{j} \overline{d w_{i}^{j}}\right) \\
\geq & i \frac{1}{h_{i}} \partial h_{i} \wedge \bar{\partial} h_{i} .
\end{aligned}
$$

Then, by Cauchy-Schwarz inequality,

$$
i h\langle\partial \bar{\partial} h ; t, \bar{t}\rangle \geq\left(\sum_{i} h_{i}\right)\left(\sum \frac{i\left\langle\partial h_{i} \wedge \bar{\partial} h_{i} ; t_{i}, \overline{t_{i}}\right\rangle}{h_{i}}\right) \geq\left(\sum\left\langle\partial h_{i} \wedge \bar{\partial} h_{i} ; t_{i}, \bar{t}_{i}\right\rangle^{1 / 2}\right)^{2} \geq i\langle\partial h \wedge \bar{\partial} h ; t, \bar{t}\rangle
$$

and Condition I is satisfied for any $s^{\prime} \geq 0$.

\subsection{Pseudo-convexity of $\widetilde{\Omega}$}

In general, even for very simple functions $h$, the domain $\widetilde{\Omega}$ is not pseudo-convex. For example, if $\Omega$ is the unit ball it is very easy to write a defining function $\rho$ of $\Omega$ such that the domain $\left\{(z, w) \in \mathbb{C}^{n} \times \mathbb{C}\right.$, s. t. $\left.\rho(z)+|w|^{2}<0\right\}$ is not pseudo-convex (see Remark 2.2).

If $\Omega$ admits a smooth defining function which is pluri-subharmonic in $\bar{\Omega}$, it suffices to take $h$ pluri-subharmonic. But this is not the general case (c.f. [DF77b]), so, we have to choose a convenient defining function for $\Omega$ :

Proposition 2.1. Let $Z \in \mathbb{C}^{n}$ and let $U$ (resp. V) be an open neighborhood of $Z$ (resp. of the origin in $\mathbb{C}^{m}$ ). Let $\rho: U \rightarrow \mathbb{R}($ resp. $h: V \rightarrow \mathbb{R}_{+}$) be a smooth function such that $\rho(Z)=0$ and $\nabla \rho$ does not vanishes in $U$ (resp $\nabla h(w) \neq 0$ if and only if $w \neq 0$ and $h(w) \neq 0$ if $w \neq 0)$. Assume that there exist $s \in] 0,1]$ and $s^{\prime} \in\left[0, s\left[\right.\right.$ such that $-(-\rho)^{s}$ is strictly pluri-subharmonic in the open set $G=\{z \in U$ s. t. $\rho(z)<0\}$ and $h^{s^{\prime}}$ is pluri-subharmonic in $V$. Let $\partial \widetilde{G}$ be the set

$$
\partial \widetilde{G}=\{(z, w) \in U \times V \text { s. t. } r(z, w)=\rho(z)+h(w)=0\} .
$$

Then, if not empty $\partial \widetilde{G}$ is a smooth hypersurface, and, at every point $\left(z^{1}, w^{1}\right) \in \partial \widetilde{G}$ :

(1) The restriction of $i \partial \bar{\partial} r$ to the complex tangent space of $\partial \widetilde{G}$ at $\left(z^{1}, w^{1}\right)$ is non-negative.

(2) If $w^{1} \neq 0$, the restriction of $i \partial \bar{\partial} r$ to the complex tangent space of $\partial \widetilde{G}$ at $\left(z^{1}, w^{1}\right)$ is positive definite if $h$ is strictly pluri-subharmonic at $w^{1}$. 
Proof. Note that, since the gradient of $r$ does not vanishes on $U \times V, \partial \widetilde{G}$ is a smooth hypersurface if it is not empty. Moreover, $s^{\prime}$ being $\leq 1, h$ is pluri-subharmonic on $V$. The hypothesis on $\rho$ is

$$
i \partial \bar{\partial} \rho \geq i \frac{1-s}{\rho} \partial \rho \wedge \bar{\partial} \rho+\varepsilon i \partial \bar{\partial}|z|^{2},
$$

where $\varepsilon$ is a non-negative function, strictly positive in $G$.

Let $\left(z^{1}, w^{1}\right)$ be a point of $\partial \widetilde{G}$ and $t=\left(t_{z}, t_{w}\right) \in \mathbb{C}^{n} \times \mathbb{C}^{m}$ be a vector of the complex tangent space of $\partial \widetilde{G}$ at $\left(z^{1}, w^{1}\right)$.

If $w^{1}=0$, as the hypotheses on $h$ imply $\nabla h(0)=0, t_{z}$ is "tangent" to $\rho$ at $z^{1}$ (i.e. $\left\langle\partial \rho, t_{z}\right\rangle\left(z^{1}\right)=0$ ). Thus the inequality (2.3) and the pluri-subharmonicity of $h$ imply $\langle i \partial \bar{\partial} r ; t, \bar{t}\rangle \geq 0$ showing (1).

Suppose now $w^{1} \neq 0$. Note that, $t$ being tangent to $r$, we have $\sum_{i} \frac{\partial \rho}{\partial z_{i}}\left(z^{1}\right) t_{z}^{i}=-\sum_{j} \frac{\partial h}{\partial w_{j}}\left(w^{1}\right) t_{w}^{j}$, and $\rho\left(z^{1}\right)=-h\left(w^{1}\right)$. Then (2.3) gives

$$
\left\langle i \partial \bar{\partial} \rho\left(z^{1}\right) ; t_{z}, \overline{t_{z}}\right\rangle \geq \frac{1-s}{-h\left(w^{1}\right)}\left|\sum_{j} \frac{\partial h}{\partial w_{j}}\left(w^{1}\right) t_{w}^{j}\right|^{2}+\varepsilon\left|t_{z}\right|^{2} .
$$

Thus

$$
\left\langle i \partial \bar{\partial} r\left(z^{1}, w^{1}\right) ; t, \bar{t}\right\rangle \geq \frac{1-s}{-h\left(w^{1}\right)}\left|\sum_{j} \frac{\partial h}{\partial w_{j}}\left(w^{1}\right) t_{w}^{j}\right|^{2}+\left\langle i \partial \bar{\partial} h\left(w^{1}\right) ; t_{w}, \overline{t_{w}}\right\rangle+\varepsilon\left|t_{z}\right|^{2} .
$$

The conclusion comes then from the fact that the hypothesis made on $h$ is

$$
i \partial \bar{\partial} h \geq \max \left\{i \frac{1-s^{\prime}}{h} \partial h \wedge \bar{\partial} h, \varepsilon_{1} i \partial \bar{\partial}|w|^{2}\right\}
$$

with $0 \leq s^{\prime}<s$ and $\varepsilon_{1}$ a non-negative function, which is positive at $w^{1}$ if $h$ is strictly pluri-subharmonic at that point: $s^{\prime} \leq s$ gives immediately (1), and, if $h$ is strictly pluri-subharmonic at $w^{1}$, then $\varepsilon\left(z^{1}\right)>0, \varepsilon_{1}\left(w^{1}\right)>0$ and

$$
\left\langle i \partial \bar{\partial} r\left(z^{1}, w^{1}\right) ; t, \bar{t}\right\rangle \geq \varepsilon_{2}|t|^{2},
$$

for $\varepsilon_{2}>0$ small.

Proposition 2.2. Let $\Omega$ be a bounded pseudo-convex domain, with smooth boundary. Let $h$ be a smooth non-negative function defined in $\mathbb{C}^{m}$. Let $\rho=\rho_{s}$ be a smooth defining function of $\Omega$ satisfying (2.2) as stated in Section 2.1.

(1) Then, if h satisfies Condition I of Section 2.2, $\widetilde{\Omega}$ is pseudo-convex.

(2) Moreover, for $\left(z^{0}, w^{0}\right) \in \partial \widetilde{\Omega}, w^{0} \neq 0$ if, in addition, $h$ is strictly pluri-subharmonic at $w^{0}, \widetilde{\Omega}$ is strictly pseudo-convex at $\left(z^{0}, w^{0}\right)$.

Proof. Let $\left(z^{0}, w^{0}\right) \in \partial \widetilde{\Omega}$. If $\partial \rho\left(z^{0}\right) \neq 0, \widetilde{\Omega}$ is pseudo-convex at $\left(z^{0}, w^{0}\right)$ by Proposition 2.1. If $\partial \rho\left(z^{0}\right)=0$, then $z^{0} \in \Omega$ and (2.3) shows that $\rho$ is strictly pluri-subharmonic in a neighborhood of $z^{0}$. Thus $r$ is pluri-subharmonic in a neighborhood of $\left(z^{0}, w^{0}\right)$ and strictly pluri-subharmonic if $h$ is strictly pluri-subharmonic at $w^{0}$.

Remark 2.2. When $m=1$ and $h(w)=|w|^{2}$, the domain $\widetilde{\Omega}=\{\rho(z)+h(w)<0\}$ is pseudo-convex at a point $\left(z^{1}, w^{1}\right) \in \partial \widetilde{\Omega}$, $w^{1} \neq 0$, if and only if

$$
i \partial \bar{\partial} \rho\left(z^{1}\right) \geq \frac{i}{\rho\left(z^{1}\right)}(\partial \rho \wedge \bar{\partial} \rho)\left(z^{1}\right) .
$$

For example, if $\rho$ is the signed distance to the boundary of $\Omega$, by Oka's theorem, $\left\{\rho(z)+|w|^{2}<0\right\}$ is pseudo-convex.

Example 2.2. With the function $h$ given in Example 2.1, (1) of Proposition 2.2 applies for any pseudo-convex domain $\Omega$. Note also that, $\widetilde{h_{1}}(w)=h(w)+|w|^{2 q}, q \in \mathbb{N}_{*}$ satisfies the condition stated in (2) of the proposition.

Proof. As noted in the proof of Example 2.1,i $\bar{\partial}\left(|w|^{2 q}\right) \geq \frac{i}{|w|^{2 q}} \partial\left(|w|^{2 q}\right) \wedge \bar{\partial}\left(|w|^{2 q}\right)$.

Remark 2.3. For $\Omega$ pseudo-convex in $\mathbb{C}^{n}$, E. Ligocka considered, in [Lig89], the domains

$$
\widetilde{\Omega}_{N, k}=\left\{(z, w) \in \mathbb{C}^{n} \times \mathbb{C}^{m} \text { s. t. } \rho(z)+|w|^{2 N k}<0\right\},
$$

where $k$ is a sufficiently large integer such that the defining function $\rho$ of $\Omega$ satisfies that $-(-\rho)^{1 / k}$ is strictly plurisubharmonic in $\Omega$ and $N$ a positive integer. She showed that if $\Omega$ is "weakly regular", then so is $\widetilde{\Omega}_{N, k}$. 


\subsection{Type finiteness of $\widetilde{\Omega}$}

We now investigate the question concerning the type of the domain $\widetilde{\Omega}$ defined by equation (2.1) with a defining function $\rho$ of $\Omega$ which does not necessarily satisfy (2.2).

Let us introduce first a notation. If $g$ is a real or complex valued smooth function defined in a neighborhood of the origin in $\mathbb{R}^{d}$, we call the order of $g$ at the origin the integer $\operatorname{ord}_{0}(g)$ defined by $\operatorname{ord}_{0}(g)=\infty$ if $g^{(\alpha)}(0)=0$ for all multi-index $\alpha \in \mathbb{N}^{d}$ and

$$
\operatorname{ord}_{0}(g)=\min \left\{k \in \mathbb{N} \text { such that there exists } \alpha \in \mathbb{N}^{d},|\alpha|=\sum \alpha_{i}=k \text { such that } g^{(\alpha)}(0) \neq 0\right\}
$$

otherwise. Moreover if $f=\left(f_{1}, \ldots, f_{q}\right)$ is a smooth function defined in a neighborhood of the origin in $\mathbb{R}^{d}$ with values in $\mathbb{R}^{q}$ the order of $f$ at the origin is $\operatorname{ord}_{0}(f)=\min \left\{\operatorname{ord}_{0}\left(f_{i}\right), 1 \leq i \leq q\right\}$. If $h$ is a smooth function defined in a neighborhood of the origin in $\mathbb{C}^{m}$, then, for all function $\varphi$ from the unit disc of the complex plane into $\mathbb{C}^{m}$ such that $\varphi(0)=0, h \circ \varphi$ is smooth in a neighborhood of the origin in $\mathbb{C}$. Then we call the type of $h$ at the origin the supremum of $\frac{\operatorname{ord}_{0}(h \circ \varphi)}{\operatorname{ord}_{0}(\varphi)}$, taken over all non-zero holomorphic function $\varphi$ from the unit disc of the complex plane into $\mathbb{C}^{m}$ such that $\varphi(0)=0$. If this supremum is finite, we will say that $h$ is of finite type at the origin and we will denote this supremum by typ $\operatorname{p}_{0}(h)$. Moreover, if $k$ is a smooth function defined in a neighborhood of a point $z_{0} \in \mathbb{C}^{m}$, the type typ $z_{0}(k)$ of $k$ at $z_{0}$ is typ $\operatorname{p}_{0}\left(h_{k}\right)$ where $h_{k}(z)=k\left(z_{0}+z\right)$ and we say that $k$ is of finite type at $z_{0}$ if $\operatorname{typ}_{z_{0}}(k)<+\infty$.

Proposition 2.3. Let $z_{0} \in \mathbb{C}^{n}$ and $U$ (resp. V) be an open neighborhood of $z_{0}$ (resp. of the origin in $\mathbb{C}^{m}$ ). Let $\rho: U \rightarrow \mathbb{R}($ resp. $\left.h: V \rightarrow \mathbb{R}_{+}\right)$be a smooth function such that $\rho\left(z_{0}\right)=0$ and $\nabla \rho$ does not vanishes in $U($ resp $\nabla h(w) \neq 0$ if $w \neq 0$ and $h(w) \neq 0$ if and only if $w \neq 0)$. Assume that the restriction of $i \partial \bar{\partial} \rho$ to the complex tangent space to the hypersurface $\partial G=\{z \in U$ s. $t . \rho(z)=0\}$ is non-negative, that $\partial G$ is of finite type $\tau$ at the point $z_{0}$ and that $h$ is of finite type typ $\mathrm{p}_{0}(h)$ at the origin.

Then the boundary of $\widetilde{G}=\{(z, w) \in U \times V$ s. $t . r(z, w)=\rho(z)+h(w)<0\}$ is of finite type $\max \left(\tau, \operatorname{typ}_{0}(h)\right)$ at the point $\left(z_{0}, 0\right)$.

Proof. To simplify the notations, we can assume $z_{0}=0$. Let $\Phi=(\varphi, \psi)$ be a non-constant holomorphic function from the unit disc of the complex plane into $U \times V$ such that $\Phi(0)=0$. We want to estimate $\frac{\operatorname{ord}_{0}(r \circ \Phi)}{\operatorname{ord}_{0}(\Phi)}$, and therefore we can assume that the first derivative of $r \circ \Phi$ vanishes at the origin. As the gradient of $h$ vanishes at the origin, this implies that the first derivative of $\rho \circ \varphi$ vanishes at the origin which means that the gradient of $\varphi$ at 0 is "tangent" to $\rho$ at 0 (i.e. $\sum \frac{\partial \varphi_{i}}{\partial \zeta} \frac{\partial \rho}{\partial z_{i}}(0)=0$ ). Clearly, by the hypothesis made on $\partial G$, we can assume that the order of $h \circ \psi$ at the origin is not infinite, and then, by Lemma 8.1 of [CD08] and the hypothesis made on $h$, there exists an integer $k \geq 1$ such that all the derivatives of order $<2 k$ of $h \circ \psi$ vanish at the origin and $\triangle^{k}(h \circ \psi)(0)>0$.

As $\operatorname{ord}_{0}(\Phi)$ is the minimum of $\operatorname{ord}_{0}(\varphi)$ and $\operatorname{ord}_{0}(\psi)$, to prove the proposition it suffices to prove that

$$
\operatorname{ord}_{0}(r \circ \Phi) \leq \min \left\{\operatorname{ord}_{0}(\rho \circ \varphi), \operatorname{ord}_{0}(h \circ \psi)\right\}=\min \left\{\operatorname{ord}_{0}(\rho \circ \varphi), 2 k\right\}
$$

(note that this not a trivial consequence of the fact that $\rho$ and $h$ are decoupled).

Note first that, if $\operatorname{ord}_{0}(\rho \circ \varphi)<2 k$ or $\operatorname{ord}_{0}(\rho \circ \varphi)>2 k$, the inequality is obvious. Thus we can assume ord $0(\rho \circ \varphi)=2 k$, and we have to prove that $\operatorname{ord}_{0}(r \circ \Phi)=2 k$.

Suppose it is not the case: it follows that all the derivatives of $r \circ \Phi$ of order $\leq 2 k$ vanish at 0 . Consider $\triangle^{k}(\rho \circ \varphi)(0)$. As $\varphi$ is holomorphic, we have

$$
\triangle^{k}(\rho \circ \varphi)(0)=\triangle^{k-1}\left(\left\langle\partial \bar{\partial} \rho(\varphi) ; \varphi^{\prime}, \overline{\varphi^{\prime}}\right\rangle\right)(0) .
$$

As all the derivatives of $\rho \circ \varphi$ of order less or equal than $2 k-1$ vanish at the origin, we have $\rho \circ \varphi(\zeta)=\mathrm{O}\left(\zeta^{2 k}\right)$. For $\zeta$ small, let $\xi=\xi(\zeta)$ be the projection of $\varphi(\zeta)$ on $\{z \in U$ s. t. $\rho(z)=0\}$ so that $\varphi(\zeta)-\xi=\mathrm{O}\left(\zeta^{2 k}\right)$. By the hypothesis ord $0(\rho \circ \varphi)=2 k$, all the derivatives of $\left\langle\partial \rho(\varphi) ; \varphi^{\prime}\right\rangle$ of order less or equal than $2 k-2$ vanish at the origin, which implies that there exists a vector $T=T(\zeta)$ tangent to $\{\rho=\rho(\xi)\}$ at the point $\xi$ such that $\varphi^{\prime}(\zeta)=T+\mathrm{O}\left(\zeta^{2 k-1}\right)$. Then, by hypothesis on the hypersurface $\partial G$, we have

$$
\left\langle i \partial \bar{\partial} \rho(\varphi(\zeta)) ; \varphi^{\prime}(\zeta), \overline{\varphi^{\prime}(\zeta)}\right\rangle=\langle i \partial \bar{\partial} \rho(\xi) ; T, \bar{T}\rangle+\mathrm{O}\left(\zeta^{2 k-1}\right) \geq-C|\zeta|^{2 k-1} .
$$

Applying Lemma 8.1 of [CD08] to the positive function

$$
\left\langle i \partial \bar{\partial} \rho(\varphi(\zeta)) ; \varphi^{\prime}(\zeta), \overline{\varphi^{\prime}(\zeta)}\right\rangle+C|\zeta|^{2 k-1}
$$

by (2.4), we get $\triangle^{k}(\rho \circ \varphi)(0) \geq 0$ which is impossible since $0=\triangle^{k}(r \circ \Phi)(0)=\triangle^{k}(\rho \circ \varphi)(0)+\triangle^{k}(h \circ \psi)(0)>0$.

Recall that in Proposition 2.3 we do not assume that $\rho$ necessarily satisfies (2.2).

Applying this proposition to a general pseudo-convex domain, we get:

Corollary 1. Assume that $\rho=\rho_{\text {s }}$ where $\rho_{s}$ satisfy (2.2) as stated in Section 2.1.

(1) Assume that $h$ satisfies Condition I of Section 2.2. Let $z^{0}$ be a boundary point of $\Omega$. If $\partial \Omega$ is of finite type $\tau$ at $z^{0}$ and if $h$ is of finite type $\operatorname{typ}_{0}(h)$ at the origin, then $\partial \widetilde{\Omega}$ is of finite type $\max \left(\tau, \operatorname{typ}_{0}(h)\right)$ at the point $\left(z^{0}, 0\right)$. Moreover, if $h$ is strictly pluri-subharmonic in $\mathbb{C}^{n} \backslash\{0\}$ then $\partial \widetilde{\Omega}$ is strictly pseudo-convex at every boundary point $\left(z^{0}, w^{0}\right)$ such that $w^{0} \neq 0$. 
(2) Assume that $h$ satisfy Condition II of Section 2.2. Then $\widetilde{\Omega}$ is pseudo-convex and, at every point $\left(z^{0}, w^{0}\right) \in \partial \widetilde{\Omega}, w^{0} \neq 0$, $\partial \widetilde{\Omega}$ is of finite type $\max _{i}$ s. $t$. $w_{i}^{0}=0\left\{\operatorname{typ}_{0}\left(h_{i}\right)\right\}$ if there exists some $i$ such that $w_{i}^{0}=0$ and is strictly pseudo-convex if $w_{i}^{0} \neq 0$ for all $i$.

Proof. The first part of (1) is a special case of Proposition 2.3, and the second part is stated in Proposition 2.2.

Let us now prove (2). The pseudo-convexity of $\widetilde{\Omega}$ follows from the results of Section 2.3 . If $w_{i}^{0} \neq 0$ for all $i$, as before, $\partial \widetilde{\Omega}$ is strictly pseudo-convex at $\left(z^{0}, w^{0}\right)$. So, assume that there exists some $i$ such that $w_{i}^{0}=0$. Without loss of generality, we can suppose that $w_{k+1}^{0}=\ldots=w_{p}^{0}=0, k<p$, and $w_{l}^{0} \neq 0$ for $1 \leq l \leq k$. Let us denote $w=\left(w^{\prime}, w^{\prime \prime}\right)$, with $w^{\prime}=\left(w_{1}, \ldots, w_{k}\right)$, $w^{\prime \prime}=\left(w_{k+1}, \ldots, w_{p}\right)$, and $\rho_{1}\left(z, w^{\prime}\right)=\rho(z)+\sum_{i=1}^{k} h_{i}\left(w_{i}\right)$. In a neighborhood of $\left(z^{0}, w^{\prime 0}\right), \nabla \rho_{1}$ does not vanish, $\left\{\rho_{1}<0\right\}$ is strictly pseudo-convex, and we can apply Proposition 2.3 to the domain $\rho_{1}\left(z, w^{\prime}\right)+\sum_{i=k+1}^{p} h_{i}\left(w_{i}\right)<0$ and the function $h_{1}\left(w^{\prime \prime}\right)$ at the point $\left(z^{0}, w^{\prime 0}, 0\right)$.

When $\Omega$ admits a pluri-subharmonic defining function which is of finite type (in the sense defined at the beginning of the section) everywhere in $\bar{\Omega}$, the proposition gives:

Corollary 2. Assume $\Omega$ admits a defining function $\rho$ pluri-subharmonic in a neighborhood of $\bar{\Omega}$ and of finite type in $\bar{\Omega}$. Assume that h satisfies Condition II of Section 2.2. Then the domain $\widetilde{\Omega}$, defined with $\rho$, is pseudo-convex of finite type. More precisely, at every point $\left(z^{1}, w^{1}\right) \in \partial \widetilde{\Omega}$ the type of $\partial \widetilde{\Omega}$ is bounded by $\max \left\{\operatorname{typ}_{z^{1}}(\partial \Omega), \operatorname{typ}_{0}\left(h_{i}\right), 1 \leq i \leq p\right\}$ if $w^{1}=0$ and by

$$
\max \left\{2 \operatorname{typ}_{z^{1}}(\rho), \max _{\text {i such that } w_{i} \neq 0}\left\{\operatorname{typ}_{0}\left(h_{i}\right)\right\}\right\}
$$

otherwise.

Proof. Let us first consider the case $p=1$. By Proposition $2.3, \widetilde{\Omega}$ is of finite type $\max \left\{\operatorname{typ}_{z^{1}}(\partial \Omega)\right.$, typ $\left._{0}(h)\right\}$ at every point $\left(z^{1}, 0\right) \in \partial \widetilde{\Omega}$, and we have to study the finiteness at points $\left(z^{1}, w^{1}\right) \in \partial \widetilde{\Omega}$ such that $w^{1} \neq 0$. Let $\Phi=(\varphi, \psi)$ be a non-constant holomorphic function from the unit disc of the complex plane into a neighborhood of $\left(z^{1}, w^{1}\right)$ such that $\Phi(0)=\left(z^{1}, w^{1}\right)$. We have to estimate $\tau_{\Phi}=\frac{\operatorname{ord}_{0}(r \circ \Phi)}{\operatorname{ord}_{0}(\Phi-\Phi(0))}$, and we can, of course, assume that $\operatorname{ord}_{0}(r \circ \Phi) \geq 2$.

Let $k$ be a positive integer and let us assume that all the derivatives of order $\leq 2 k$ of $r \circ \Phi$ vanish at the origin. Then

$$
\triangle(r \circ \Phi)(0)=\left\langle\partial \bar{\partial} \rho ; \varphi^{\prime}, \overline{\varphi^{\prime}}\right\rangle(0)+\left\langle\partial \bar{\partial} h ; \psi^{\prime}, \overline{\psi^{\prime}}\right\rangle(0)=0,
$$

and the hypothesis on $\rho$ and $h$ (pluri-subharmonicity of $\rho$ and strict pluri-subharmonicity of $h)$ imply $\left\langle\partial \bar{\partial} \rho ; \varphi^{\prime}, \overline{\varphi^{\prime}}\right\rangle(0)=$ $\left\langle\partial \bar{\partial} h ; \psi^{\prime}, \overline{\psi^{\prime}}\right\rangle(0)=0$ and the last equality implies $\psi^{\prime}(0)=0$. Moreover Lemma 8.1 of [CD08] implies that, for $1 \leq j \leq k-1$, $\triangle^{j}\left(\left\langle\partial \bar{\partial} h ; \psi^{\prime}, \overline{\psi^{\prime}}\right\rangle\right)(0)=0$, and, by induction, a simple calculation shows that this implies $\psi^{(j+1)}(0)=0,1 \leq j \leq k-1$. Then all the derivatives of order $\leq k$ of $h \circ \psi$ vanish at the origin, and, thus, the same is true for the derivatives of $\rho \circ \varphi$.

This implies first that the order of $r \circ \Phi$ cannot be infinite at 0 . Assume it is $l$, and let $k$ be a positive integer such that $l=2 k+1$ or $l=2 k+2$. In both cases, the orders of $\rho \circ \varphi-\rho\left(z^{1}\right), h \circ \varphi-h\left(w^{1}\right)$ and $\psi-\psi(0)$ are $\geq k+1$ and we have $\frac{\operatorname{ord}_{0}(r \circ \Phi)}{\operatorname{ord}_{0}(\Phi-\Phi(0))} \leq \frac{2 \operatorname{ord}_{0}\left(\rho \circ \varphi-\rho\left(z^{1}\right)\right)}{\operatorname{ord}_{0}(\Phi-\Phi(0))}$ which implies $\tau_{\Phi} \leq 2$ if $\operatorname{ord}_{0}(\varphi-\varphi(0)) \geq k+1$ and $\tau_{\Phi} \leq 2 \operatorname{typ}_{z^{1}}(\rho)$ if not.

The case $p \geq 2$ follows easily. If, for all $i, 1 \leq i \leq p, w_{i}^{1} \neq 0$ then $h$ is strictly pluri-subharmonic at $w^{1}$ and the previous proof applies. Otherwise, to simplify notations, we can assume that $w_{i}^{1} \neq 0$ for $1 \leq i \leq r<p$ and $w_{i}^{1}=0$ for $r+1 \leq i \leq$ $p$. Denoting $u=\left(w_{1}, \ldots w_{r}\right), v=\left(w_{r+1}, \ldots, w_{p}\right), \rho_{1}(z, u)=\rho(z)+\sum_{i=1}^{r} h_{i}\left(w_{i}\right)$ and $h_{1}(v)=\sum_{i=r+1}^{p} h_{i}\left(w_{i}\right)$, the previous case shows that $\rho_{1}$ is pluri-subharmonic and the type of $\rho_{1}$ at $\left(z^{1}, u^{1}\right)$ is bounded by $2 \operatorname{typ}_{z^{1}}(\rho)$. The conclusion is obtained applying Proposition 2.3.

\subsection{Geometric separation}

If the domain $\Omega$ is completely geometrically separated at a boundary point $z_{0}$ (see [CD08] for definition), we do not know, in general, if $\widetilde{\Omega}$ has the same property at the point $\left(z_{0}, 0\right)$. We can only prove the weaker following result (for which we will not give a proof because we do not have any application):

Proposition 2.4. Assume that $\Omega$ is of finite type at $z_{0} \in \partial \Omega$ and that $h(w)=\sum\left|w_{i}\right|^{2 q_{i}}, w_{i} \in \mathbb{C}$. Then for all Diederich-Fornass defining function $\rho$ of $\Omega$ of the form $\rho=\sigma e^{-L|z|^{2}}$ (see [DF77a]) with L large enough (depending only on $\Omega$ ), we have:

if there exist a neighborhood $V$ of $z_{0}, K>0$ and a finite dimensional vector space $E_{0}$ of $(1,0)$ vector fields tangent to $\rho$ in $V$ (i.e. $L \rho \equiv 0$ for $L \in E_{0}$ ) such that, at any point of $V \cap \Omega$ and for any $\delta>0$ there exists a $(K, \delta)$-extremal basis for $\rho$ whose elements belong to $E_{0}$, then $\widetilde{\Omega}$ is geometrically separated at $\left(z_{0}, 0\right)$.

Note that the hypothesis in this proposition is stronger than the simple fact that $\partial \Omega$ is geometrically separated at $z_{0}$ : the existence of extremal basis is assumed not only on the points of $\partial \Omega \cap V$ but on all $\Omega \cap V$ (condition which depends not only on $\Omega$ but also on the choice of $\rho$ ). Unfortunately, if we add the hypothesis that all the level set of $\rho$ are "completely geometrically separated" in $\Omega \cap V$ we can not prove, in general, that $\widetilde{\Omega}$ has the same property at $\left(z_{0}, 0\right)$. The only general result we have is when $\Omega$ is in $\mathbb{C}^{2}$ (see Remark 2.4): 
Theorem 2.1. Assume $\Omega$ is pseudo-convex of finite type in $\mathbb{C}^{2}$. Assume that $\rho=\rho_{s}$ with $\rho_{s}$ satisfying (2.2) as stated in Section 2.1 and that $h$ satisfies Condition III of Section 2.2. Then the domain

$$
\widetilde{\Omega}=\left\{(z, w)=\left(z, w_{1}, \ldots, w_{m}\right) \in \mathbb{C}^{2} \times \mathbb{C}^{m} \text { s. } t . r(z, w)=\rho(z)+\sum_{i=1}^{m} h_{i}\left(w_{i}\right)<0\right\}
$$

is pseudo-convex of finite type and has a Levi form which is locally diagonalizable at every point of its boundary. In particular $\widetilde{\Omega}$ is completely geometrically separated (c.f. [CD08]).

Proof. Let $\left(z^{0}, w^{0}\right)$ be a boundary point of $\widetilde{\Omega}$. If $w_{i}^{0} \neq 0$ for all $i$, then, by (2) of Corollary 1 following Proposition $2.3, \partial \widetilde{\Omega}$ is strictly pseudo-convex at $\left(z^{0}, w^{0}\right)$. Thus we have only two cases to consider:

(1) $w^{0}=0$;

(2) there exist $i$ and $j$ such that $w_{i}^{0}=0$ and $w_{j}^{0} \neq 0$.

Let us consider the first case. Denote by $L$ (resp. $N$ ) a non-vanishing vector field "complex tangent" (resp. normal) to $\rho$ in a neighborhood of $z^{0}$ (i.e. $L$ is of type $(1,0)$ and $L \rho \equiv 0$ ). We assume that $N$ is chosen so that $N \rho \equiv 1$ in that neighborhood. Without changing the notation, we will consider these vector fields defined in a neighborhood of $\left(z^{0}, 0\right)$ so that $L r \equiv 0$ and $N r \equiv 1$ in this neighborhood. Let us define $m$ vector fields, $Z_{i}$, "complex tangent" to $r$, in a neighborhood of $\left(z^{0}, 0\right)$ by

$$
Z_{i}=\frac{\partial}{\partial w_{i}}-\frac{\partial h_{i}}{\partial w_{i}} N
$$

and then $m$ new vector fields, $W_{i}$, also "complex tangent" to $r$, by

$$
\begin{aligned}
W_{1} & =Z_{1}, \\
W_{k+1} & =Z_{k+1}-\sum_{j=1}^{k} a_{k+1}^{j} W_{j} \text { for } k \geq 2 .
\end{aligned}
$$

We now show, by induction over $k$, that it is possible to choose the coefficients $a_{k}^{j}$ so that the coefficient of the Levi form of $r,\left[W_{k}, \overline{W_{k^{\prime}}}(\partial r)\right.$, vanishes identically on the neighborhood of $\left(z^{0}, 0\right)$. To simplify notations, in this proof, the character $*$ will denote a $\mathscr{C}^{\infty}$ function in a neighborhood of the origin.

Suppose that the vector fields $W_{i}, 1 \leq i \leq k$, have been constructed with coefficients $a_{i}^{j}, 2 \leq i \leq k, 1 \leq j \leq i-1$ satisfying the two following properties:

(1) $a_{i}^{j}=* \frac{\partial h_{i}}{\partial w_{i}}$

(2) $\left[W_{i}, \overline{W_{i}}\right](\partial r)=\gamma_{i} \frac{\partial^{2} h_{i}}{\partial w_{i} \partial \overline{w_{i}}}$, where $\gamma_{i}$ is a $\mathscr{C}^{\infty}$ real function in a neighborhood of the origin of modulus greater than $1 / 2$,

and let us prove that $W_{k+1}$ can be constructed, so that the coefficients $a_{k+1}^{j}, 1 \leq j \leq k$ satisfy the above conditions. Note that the hypotheses made on $h_{i}$ imply first that (2) follows from (1) because (1) implies

$$
\left[W_{i}, \overline{W_{i}}\right](\partial r)=\frac{\partial^{2} h_{i}}{\partial w_{i} \partial \overline{w_{i}}}+\left|\frac{\partial h_{i}}{\partial w_{i}}\right|^{2}[N, \bar{N}](\partial \rho)+* \frac{\partial h_{i}}{\partial w_{i}}+* \frac{\partial h_{i}}{\partial \bar{w}_{i}} .
$$

Note also that $W_{1}$ satisfy trivially (2).

Thus we construct $W_{k+1}$ with coefficients $a_{k+1}^{j}$ satisfying (1).

For $j \leq k$, by induction, we have

$$
\left[W_{k+1}, \overline{W_{j}}\right](\partial r)=-\frac{\partial h_{j}}{\partial \overline{w_{j}}}\left[N, \overline{W_{j}}\right](\partial r)-a_{k+1}^{j}\left[W_{j}, \overline{W_{j}}\right](\partial r)
$$

with

$$
\begin{aligned}
{\left[N, \overline{W_{j}}\right](\partial r) } & =-\frac{\partial h_{j}}{\partial \overline{w_{j}}}[N, \bar{N}](\partial \rho)-\sum_{1 \leq l<j} \overline{a_{j}^{l}}\left[N, \overline{W_{l}}\right](\partial r) \\
& =* \frac{\partial h_{j}}{\partial \bar{w}_{j}}
\end{aligned}
$$

if $2 \leq j \leq k$, and

$$
\left[N, \overline{W_{1}}\right](\partial r)=* \frac{\partial h_{1}}{\partial \overline{w_{1}}} .
$$

This shows, by Condition III of $h$, that the $a_{k+1}^{j}$ can be defined satisfying (1) and such that $\left[W_{k+1}, \overline{W_{j}}\right](\partial r) \equiv 0$.

To finish the proof of the first case, we modify the vector field $L$ replacing it by $L_{1}=L-\sum_{k=1}^{m} b_{k} W_{k}$ choosing the $b_{k}$ so that the basis $\left(L_{1}, W_{1}, \ldots, W_{m}\right)$ diagonalizes the Levi form of $r$ in a neighborhood of $\left(z^{0}, 0\right)$ which means, now, $\left[L_{1}, \overline{W_{i}}\right](\partial r) \equiv 0$ in 
that neighborhood:

$$
\begin{aligned}
{\left[L_{1}, \overline{W_{i}}\right](\partial r) } & =\left[L, \overline{W_{i}}\right](\partial r)-b_{i}\left[W_{i}, \overline{W_{i}}\right](\partial r) \\
& = \begin{cases}-\frac{\partial h_{i}}{\partial \overline{w_{i}}}[L, \bar{N}](\partial r)-\sum_{1 \leq l<i} \overline{a_{i}^{l}}\left[L, \overline{W_{i}}\right](\partial r)-b_{i}\left[W_{i}, \overline{W_{i}}\right](\partial r) & \text { if } i \geq 2 \\
-\frac{\partial h_{1}}{\partial \bar{w}_{1}}[L, \bar{N}](\partial r)-b_{i}\left[W_{i}, \overline{W_{i}}\right](\partial r) & \text { if } i=1\end{cases} \\
& =* \frac{\partial h_{i}}{\partial \overline{w_{i}}}-b_{i}\left[W_{i}, \overline{W_{i}}\right](\partial r),
\end{aligned}
$$

and, by (2), $b_{i}$ can be chosen $\mathscr{C}^{\infty}$ in a neighborhood of $\left(z^{0}, 0\right)$.

Let us now consider the second case (2). To simplify the notations, we assume that $w_{i}^{0} \neq 0$ for $1 \leq i \leq m_{0}<m$ and $w_{i}^{0}=0$ for $m_{0}+1 \leq i \leq m$. We denote $w=\left(w^{\prime}, w^{\prime \prime}\right)$, with $w^{\prime}=\left(w_{1}, \ldots, w_{m_{0}}\right), w^{\prime \prime}=\left(w_{m_{0}+1}, \ldots, w_{m}\right), \rho^{1}\left(z, w^{\prime}\right)=\rho(z)+\sum_{i=1}^{m_{0}} h_{i}\left(w_{i}\right)$ and $h^{1}\left(w^{\prime \prime}\right)=\sum_{i=m_{0}+1}^{m} h_{i}\left(w_{i}\right)$.

By Proposition 2.1, in a neighborhood of $\left(z^{0}, w^{\prime 0}\right)$, the hypersurface $\left\{\rho^{1}=0\right\}$ is strictly pseudo-convex. Then, reducing eventually the neighborhood, there exists a basis of vector fields $\left(L_{1}, \ldots, L_{m_{0}-1}\right)$ "complex tangent" to $\rho^{1}$ (i.e. $\left.L_{i}\left(\rho^{1}\right) \equiv 0\right)$ which diagonalizes the Levi form of $\rho^{1}$ in that neighborhood. Let us denote by $N$ the complex normal vector field to $\rho^{1}$ in that neighborhood such that $N \rho^{1} \equiv 1$ (note that, reducing the neighborhood if necessary, we can assume that the gradient of $\rho^{1}$ does not vanishes in the neighborhood). We now consider the following $m-m_{0}$ vector fields (which are "complex tangent" to $\rho^{1}$ in the neighborhood)

$$
\begin{aligned}
W_{1} & =\frac{\partial}{\partial w_{m_{0}+1}}-\frac{\partial h_{m_{0}+1}}{\partial w_{m_{0}+1}} N-\sum_{i=1}^{m_{0}-1} a_{1}^{i} L_{i}, \\
W_{j} & =\frac{\partial}{\partial w_{m_{0}+j}}-\frac{\partial h_{m_{0}+j}}{\partial w_{m_{0}+j}} N-\sum_{i=1}^{m_{0}-1} a_{j}^{i} L_{i}-\sum_{l=1}^{j-1} b_{j}^{l} W_{l}, \text { for } j \geq 2 .
\end{aligned}
$$

To finish the proof of the theorem, we show that it is possible to choose the coefficients $a_{j}^{i}$ and $b_{j}^{l} \mathscr{C}^{\infty}$ in a neighborhood of $\left(z^{0}, w^{0}\right)$ so that the basis of vector field $\left(L_{1}, \ldots, L_{m_{0}-1}, W_{1}, \ldots, W_{m-m_{0}}\right)$ diagonalizes the Levi form of $r$ in that neighborhood. We do this using an induction argument similar to the one used in the first case: assume that the vector fields $W_{j}, 1 \leq j \leq k$ have been constructed and that their coefficients satisfy

(1) $a_{j}^{i}$ and $b_{j}^{l}=* \frac{\partial h_{m_{0}+j}}{\partial w_{m_{0}+j}}$, where $*$ is a $\mathscr{C}^{\infty}$ function in a neighborhood of $\left(z^{0}, w^{0}\right)$,

(2) $\left[W_{j}, \overline{W_{j}}\right](\partial r)=\gamma_{j} \frac{\partial^{2} h_{m_{0}+j}}{\partial w_{m_{0}+j} \partial \overline{w_{m_{0}+j}}}$, where $\gamma_{j}$ is a $\mathscr{C}^{\infty}$ real function in a neighborhood of $\left(z^{0}, w^{0}\right)$ greater, in modulus, than $1 / 2$.

As for the first case, note that (2) follows (1). Then, for $1 \leq j \leq m_{0}-1$,

$$
\left[W_{k+1}, \overline{L_{j}}\right](\partial r)=-\frac{\partial h_{m_{0}+k+1}}{\partial w_{m_{0}+k+1}}\left[N, \overline{L_{j}}\right](\partial r)-a_{k+1}^{j}\left[L_{j}, \overline{L_{j}}\right](\partial r),
$$

and, for $j \leq k$,

$$
\left[W_{k+1}, \overline{W_{j}}\right](\partial r)=-\frac{\partial h_{m_{0}+k+1}}{\partial w_{m_{0}+k+1}}\left[N, \overline{W_{j}}\right](\partial r)-b_{k+1}^{j}\left[W_{j}, \overline{W_{j}}\right](\partial r),
$$

and the results follow, noting that $\left[L_{j}, \overline{L_{j}}\right](\partial r)$ is bounded from below by a strictly positive constant in a neighborhood of $\left(z^{0}, w^{0}\right)$, and, as in the first case, that $\left[N, \overline{W_{j}}\right](\partial r)=* \frac{\partial h_{m_{0}+j}}{\partial \bar{w}_{m_{0}+j}}$, with $*$ is a $\mathscr{C}^{\infty}$ function in a neighborhood of $\left(z^{0}, w^{0}\right)$.

Example. If $h(w)=\sum h_{i}\left(w_{i}\right), w_{i} \in \mathbb{C}$, where each function $h_{i}$ is a positive radial analytic function vanishing at the origin, then the hypothesis of the theorem are verified.

The proof of the second case, shows that Theorem 2.1 is also valid if $\Omega$ is a smooth strictly pseudo-convex domain in $\mathbb{C}^{n}$ (of any dimension). Moreover, applying first the method of the second case and then the one of the first case, this is also true when the rank of the Levi form of $\rho$ is $\geq n-2$. Thus:

Theorem 2.2. Assume that $\rho=\rho_{s}$ with $\rho_{s}$ satisfying (2.2) as stated in Section 2.1 and that hatisfy Condition III of Section 2.2. If the rank of the Levi form of $\rho$ is $\geq n-2$, then $\widetilde{\Omega}$ is locally diagonalizable at every point of its boundary.

Remark 2.4. If $\Omega$ is a smooth bounded convex domain of finite type in $\mathbb{C}^{n}$ we do not know if it is always possible to choose a defining function $\rho$ and a function $h$ so that $\widetilde{\Omega}$ is "completely geometrically separated" at any boundary point (we will see in Section 4.2 that this is possible near $\{w=0\}$ ). 


\section{RELATIONS BETWEEN OPERATORS RELATED TO $\widetilde{\Omega}$ AND TO $\Omega$}

Assume that $\Omega$ is a smooth bounded pseudo-convex domain of finite type in $\mathbb{C}^{n}$, that $\rho=\rho_{s}$ is a defining function of $\Omega$ where $\rho_{s}$ satisfies (2.2) as stated in Section 2.1, and that $h$ satisfies, at least, Condition II of Section 2.2. Thus, by Corollary 1 of Proposition 2.3, the domain

$$
\widetilde{\Omega}=\left\{(z, w) \in \mathbb{C}^{n+m} \text { s. t. } \rho(z)+h(w)<0\right\},
$$

is a smooth bounded pseudoconvex domain of $\mathbb{C}^{n+m}$ of finite type.

In this section we assume that the functions $h_{i}$ defining $h$ are radial (i.e. $h(w)=\sum h_{i}\left(\left|w_{i}\right|\right)$, and, taking into account the properties of the $\bar{\partial}$-Neumann problem for $\widetilde{\Omega}$, we derive properties of solutions of the $\bar{\partial}$-equation and properties of the Bergman projections related to the weight

$$
\omega(z)=\int_{\{h(w)<-\rho(z)\}} d \lambda(w) .
$$

Suppose $f=\sum_{i=1}^{n} f_{i} d \overline{z_{i}}$ is a $(0,1)$-form on $\Omega$. Consider it as a $(0,1)$-form $\widetilde{f}$ in $\widetilde{\Omega}$. If $f$ is $\bar{\partial}$-closed, then so is $\widetilde{f}$, and if $\widetilde{u}$ is a solution of $\bar{\partial} \widetilde{u}=\widetilde{f}$ in $\widetilde{\Omega}$, then $\widetilde{u}$ is holomorphic in the variable $w$ and the function $u$ defined by $u(z)=\widetilde{u}(z, 0)$ is a solution of the equation $\bar{\partial} u=f$ in $\Omega$. Moreover, for all $\alpha \in \mathbb{N}^{n}$, denoting $D_{z}^{\alpha}=\frac{\partial^{|\alpha|}}{\partial z_{1}^{\alpha_{1}} \ldots, \partial z_{n}^{\alpha_{n}}}$, we have $D_{z}^{\alpha} u(z)=D_{z}^{\alpha} \widetilde{u}(z, 0), w \mapsto D_{z}^{\alpha} \widetilde{u}(z, w)$ is holomorphic, for any $p \in[1,+\infty], w \mapsto\left|D_{z}^{\alpha} \widetilde{u}(z, w)\right|^{p}$ is pluri-subharmonic, and, by the mean value property (the functions $h_{i}$ being radial),

and

$$
D_{z}^{\alpha} u(z)=(\omega(z))^{-1} \int_{\{h(w)<-\rho(z)\}} D_{z}^{\alpha} \widetilde{u}(z, w) d \lambda(w)
$$

Thus:

$$
\int_{\Omega}\left|D_{z}^{\alpha} u(z)\right|^{p} \omega(z) d \lambda(z) \leq \int_{\widetilde{\Omega}}\left|D_{z}^{\alpha} \widetilde{u}(z, w)\right|^{p} d \lambda(z, w) .
$$

Lemma 3.1. With the conditions and notations stated above, for any $p \in[1,+\infty]$ and any integer $t \geq 0$, denote by $L_{\omega}^{p, t}(\Omega)$ the Sobolev space of functions $g$ (resp. of $(0,1)$-forms $g=\sum_{i=1}^{n} g_{i} d \overline{z_{i}}$ ) such that, for all $\alpha \in \mathbb{N}^{n},|\alpha| \leq t, D_{z}^{\alpha} g$ belongs to the weighted $L^{p}$ space $L_{\omega}^{p}(\Omega)=L^{p}\left(\Omega, \omega(z) d \lambda(z)\right.$ ) (resp. to the weighted space $L_{(0,1), \omega}^{p, t}(\Omega)$ of $(0,1)$-forms $g$ on $\Omega$ whose coefficients $g_{i}$ belong to $\left.L_{\omega}^{p, t}(\Omega)\right)$ equipped with the norm $\|g\|_{L_{\omega}^{p, t}(\Omega)}=\sum_{|\alpha| \leq t}\left\|D_{z}^{\alpha} g\right\|_{L_{\omega}^{p}(\Omega)}\left(\right.$ resp. $\left.\|g\|_{L_{(0,1), \omega}^{p, t}(\Omega)}=\sum_{i=1}^{n} \sum_{|\alpha| \leq t}\left\|D_{z}^{\alpha} g_{i}\right\|_{L_{\omega}^{p}(\Omega)}\right)$. Then

(1) $f$ is in $L_{(0,1), \omega}^{p, t}(\Omega)$ if an only if $\widetilde{f}$ belongs to $L_{(0,1)}^{p, t}(\widetilde{\Omega})$ and, in this case, $\|f\|_{L_{(0,1), \omega}^{p, t}(\Omega)}=\|\widetilde{f}\|_{L_{(0,1)}^{p, t}(\widetilde{\Omega})}$;

(2) If $\tilde{u}$ belongs to $L^{p, t}(\widetilde{\Omega})$ then $u$ belongs to $L_{\omega}^{p, t}(\Omega)$ and $\|u\|_{L_{\omega}^{p, t}(\Omega)} \leq\|\widetilde{u}\|_{L^{p, t}(\widetilde{\Omega})}$.

Similarly, a function $u$ belongs to $L_{\omega}^{2}(\Omega)$ if and only if the function $\widetilde{u}$, defined on $\widetilde{\Omega}$ by $\widetilde{u}(z, w)=u(z)$, belongs to $L^{2}(\widetilde{\Omega})$. So, if $P^{\widetilde{\Omega}}$ denotes the Bergman projection of $\widetilde{\Omega}$ and $P_{\omega}^{\Omega}$ the Bergman projection of $\Omega$ with the weight $\omega$, as $h(w)=\sum_{i=1}^{p} h_{i}\left(\left|w_{i}\right|\right)$, $w_{i} \in \mathbb{C}^{m_{i}}$, by the mean value property we have $P_{\omega}^{\Omega}(u)(z)=P^{\widetilde{\Omega}}(\widetilde{u})(z, 0)$. Then:

Lemma 3.2. With the above notations,

(1) We have $\|u\|_{L_{\omega}^{p, t}(\Omega)}=\|\widetilde{u}\|_{L^{p, t}(\widetilde{\Omega})}$;

(2) We have $P_{\omega}^{\Omega}(u)(z)=P^{\widetilde{\Omega}}(\widetilde{u})(z, 0)$ and $\left\|P_{\omega}^{\Omega}(u)\right\|_{L_{\omega}^{p, t}(\Omega)} \leq\left\|P^{\widetilde{\Omega}}(\widetilde{u})\right\|_{L^{p, t}(\widetilde{\Omega})}$.

(3) For any $\bar{\partial}$-closed $(0,1)$-form $f \in L_{\omega}^{2}(\Omega)$, denoting $\widetilde{f}(z, w)=f(z), \bar{\partial}^{*} \mathscr{N}_{\widetilde{\Omega}}(\widetilde{f})(z, 0)$ is the solution of the equation $\bar{\partial} u=f$ orthogonal to holomorphic functions in $L_{\omega}^{2}(\Omega)$.

(4) If $K_{B, \omega}^{\Omega}\left(\right.$ resp. $\left.K_{B}^{\widetilde{\Omega}}\right)$ denotes the Bergman kernel of $\Omega$ associated to the measure $\omega(z) d \lambda(z)$ (resp. of $\widetilde{\Omega}$ associated to the Lebesgue measure), we have $K_{B, \omega}^{\Omega}(z, \zeta)=K_{B}^{\widetilde{\Omega}}((z, 0),(\zeta, 0))$.

Now, we will derive from these lemmas some simple weighted estimates on $\Omega$ when the corresponding unweighted estimates are known on $\widetilde{\Omega}$.

\subsection{Sobolev estimates for general pseudo-convex domain}

As $\widetilde{\Omega}$ is of finite type, by the fundamental result of D. Catlin ([Cat87]) the $\bar{\partial}$-Neumann problem of $\widetilde{\Omega}$ satisfies a subelliptic estimate. Then, all the associated operators map continuously the $L^{2}$ Sobolev spaces of $\widetilde{\Omega}$ into themselves.

To respect traditional notations, denote, for $t \in \mathbb{N}, W_{\omega}^{t}(\Omega)=L_{\omega}^{2, t}(\Omega)$ and $W_{(0,1), \omega}^{t}(\Omega)=L_{(0,1), \omega}^{2, t}(\Omega)$.

Lemmas 3.2 and 3.1 imply thus:

Theorem 3.1. Let $\Omega$ be a smooth bounded pseudo-convex domain of finite type in $\mathbb{C}^{n}$. Let $\rho=\rho_{s}$ be a defining function of $\Omega$ where $\rho_{s}$ satisfies (2.2) as stated in Section 2.1. Let $h$ be a smooth function on $\mathbb{C}^{m}$ satisfying Condition II of Section 2.2 the functions $h_{i}$ being radial. Then, $\omega$ being the weight defined by (3.1): 
(1) For any integer $t$, if $f$ is a $\bar{\partial}$-closed $(0,1)$-form in $W_{(0,1), \omega}^{t}(\Omega)$, then the solution to the equation $\bar{\partial} u=f$ orthogonal to holomorphic functions in $L_{\omega}^{2}(\Omega)$ satisfies $\|u\|_{W_{\omega}^{t}(\Omega)} \leq C\|f\|_{W_{(0,1), \omega}^{t}(\Omega)}$, the constant $C$ depending on $\rho$, $h$ and $t$;

(2) For any integer t, the weighted Bergman projection $P_{\omega}^{\Omega}$ maps the Sobolev space $W_{\omega}^{t}(\Omega)$ continuously into itself.

Theorem 1.2 is (2) of the following corollary:

Corollary. Let $\Omega$ and $\rho$ be as in the theorem. Let $r \geq 0$ be a rational number and $\varphi_{r}$ be the pluri-subharmonic function $\varphi_{r}=-r \log (-\rho)\left(c . f\right.$. Remark 2.1). Let us denote by $\bar{N}_{\varphi_{r}}$ the $\bar{\partial}$-Neumann operator for the weight $e^{-\varphi_{r}}$ acting on $(0,1)$-forms and by $\bar{\partial}_{\varphi_{r}}^{*} \mathscr{N}_{\varphi_{r}}^{(0,1)}$ the restriction to the space of $\bar{\partial}$-closed forms in $L_{(0,1),(-\rho)^{r}}^{2}(\Omega)$ of the operator $\bar{\partial}_{\varphi_{r}}^{*} \mathscr{N}_{\varphi_{r}}$ giving the $L_{(-\rho)^{r}}^{2}$ minimal solution of the $\bar{\partial}$-equation. Let us denote by $\mathscr{B} \varphi_{r}$ the Bergman projection of $L_{\varphi_{r}}^{2}(\Omega)$. Then:

(1) For all $t \geq 0, \bar{\partial}_{\varphi_{r}}^{*} \mathscr{N}_{\varphi_{r}}^{(0,1)}$ maps the subspace of $\bar{\partial}$-closed forms of $W_{(0,1),(-\rho)^{r}}^{t}(\Omega)$ continuously into $W_{(-\rho)^{r}}^{t}(\Omega)$.

(2) For all real number $t, \mathscr{B}_{\varphi_{r}}$ maps $W_{(-\rho)^{t}}^{t}(\Omega)$ continuously into itself.

Remark 3.1.

(1) In the corollary, the function $h$ is equal to $\sum\left|w_{i}\right|^{2 q_{i}}, w_{i} \in \mathbb{C}$, the integers $q_{i}$ being chosen so that $r=\sum \frac{1}{q_{i}}$. $\widetilde{\Omega}$ being of finite type, there is a gain in the Sobolev scale for the estimates of the $\bar{\partial}$-Neumann problem on $\widetilde{\Omega}$. This implies a similar gain for $\bar{\partial}_{\varphi_{r}}^{*} \mathscr{N}_{\varphi_{r}}^{(0,1)}$. But this gain is the inverse on the type of $\widetilde{\Omega}$ which is given in Corollary 1 of Proposition 2.3 and, then, can be very small depending on $r$.

(2) If $\Omega$ is a smooth bounded pseudo-convex domain in $\mathbb{C}^{n}$ (not assumed of finite type) admitting a defining function $\rho$ which is pluri-subharmonic in $\bar{\Omega}$ then:

(a) If $h$ is a positive pluri-subharmonic function satisfying $\nabla h(w) \neq 0$ if $w \neq 0, \lim _{|w| \rightarrow+\infty} h(w)=+\infty$ and $h(w)=$ $\sum_{i=1}^{p} h_{i}\left(\left|w_{i}\right|\right), w=\left(w_{1}, \ldots, w_{p}\right), w_{i} \in \mathbb{C}^{m_{i}}$, then $\widetilde{\Omega}$ is bounded, admits a pluri-subharmonic defining function and, applying a theorem of H. Boas \& E. Straube ([BS91]), we get that, for all real number $t$, the weighted Bergman projection $P_{\omega}^{\Omega}$ maps the Sobolev spaces $W_{\omega}^{t}(\Omega)$ continuously into themselves.

(b) Moreover, if $\rho$ is of finite type in $\bar{\Omega}$ then Corollary 2 of Proposition 2.3 shows that the results of the theorem and the corollary are also valid using $\rho$ to define $\widetilde{\Omega}$ and thus for other weights $\omega$.

\subsection{Lipschitz estimates for domains in $\mathbb{C}^{2}$}

Here we obtain estimates on weighted Bergman projections of $\Omega$ using only properties of the Bergman projection of the domain $\widetilde{\Omega}$. For this we need $\widetilde{\Omega}$ to be "completely geometrically separated" and we assume:

$\Omega$ is a domain in $\mathbb{C}^{2}$, or the rank of the Levi form of $\partial \Omega$ is $\geq n-2, \rho=\rho_{s}$ with $\rho_{s}$ satisfying (2.2) as stated in Section 2.1 and $h$ satisfies Condition III of Section 2.2. We denote by $\omega=\omega_{\rho, h}$ the associated weight (equation (3.1)).

Let $M$ be the type of $\Omega$. By [CD06b, CD06a], we know that $P^{\widetilde{\Omega}}$ maps continuously the Lipschitz space $\Lambda_{\alpha}(\widetilde{\Omega}), \alpha \geq 0$, into itself and that the space of holomorphic functions in $\Lambda_{\alpha}(\Omega)$ is continuously embedded in the anisotropic Lipschitz space $\Gamma_{\alpha}(\Omega)$ for $\alpha<1 / M$. Then Lemma 3.2 gives immediately:

Theorem 3.2. In the conditions stated above for $\Omega, \rho, h$ and $\omega$, the weighted Bergman projection $P_{\omega}^{\Omega}$ maps the Lipschitz space $\Lambda_{\alpha}(\Omega)$ continuously into itself for all $\alpha \geq 0$ and into the anisotropic Lipschitz space $\Gamma_{\alpha}(\Omega)$ for $\alpha<1 / M$.

Remark.

(1) In the next section, using pointwise estimates of the kernel of $P_{\omega}^{\Omega}$ we will extend the Lipschitz estimate for $P_{\omega}^{\Omega}$ to convex domains of finite type in $\mathbb{C}^{n}$ but for a smaller class of weights $\omega$.

(2) In the conditions of the preceding theorem, choosing $h(w)=\sum_{i}\left|w_{i}\right|^{q_{i}}, w_{i} \in \mathbb{C}$, the weight $\omega$ is equal to (a constant times) $(-\rho)^{q}$, with $q=\sum \frac{1}{q_{i}}$. Using that the Bergman projection $P^{\widetilde{\Omega}}$ of $\widetilde{\Omega}$ maps the Sobolev spaces $L_{s}^{p}(1<p<+\infty, s \in \mathbb{N})$ continuously into themselves ([CD06b, CD06a]), we get immediately that the weighted Bergman projections $P_{\omega}^{\Omega}$ maps the (weighted) Sobolev spaces $L_{S}^{p}\left((-\rho)^{q} d \lambda\right)$ continuously into themselves and it is easy to extended this to the spaces $L_{S}^{p}\left((-\rho)^{t} d \lambda\right)$ for $-1<t-q<p-1$.

In the next section, establishing pointwise estimates of the kernel of $P_{\omega}^{\Omega}$ we will get the (better) results stated in Theorem 1.1 .

\section{SHARP ESTIMATES OF THE WEIGHTED BERGMAN KERNEL AND PROOF OF THEOREM 1.1}

The aim of this section is to establish precise pointwise estimates of the kernel of the weighted Bergman projection $P_{\omega}^{\Omega}$ in terms of the geometry of $\Omega$ (from which we will deduce Theorem 1.1) using pointwise estimates of the kernel of $P^{\widetilde{\Omega}}$ and Lemma 3.2. Hence we need, at least, that the domain $\widetilde{\Omega}$ is "completely geometrically separated" near the set $\{(z, 0) \in \widetilde{\Omega}\}$ and to have a precise comparison of the geometries of $\widetilde{\Omega}$ and $\Omega$. We are able to do this in the two following cases:

- $\Omega$ is a finite type domain in $\mathbb{C}^{2}, \rho=\rho_{s}$ satisfies (2.2) and $h$ satisfies Condition IV of Section 2.2

- $\Omega$ is a convex domain of finite type in $\mathbb{C}^{n}, \rho=g^{4} e^{1-1 / g}-1$ where $g$ is a gauge function of $\Omega$, so that $\rho$ is convex and of finite type in a neighborhood of $\partial \Omega$, and $h$ satisfies Condition V of Section 2.2 
(the weight $\omega$ being given by (3.1)). Recall that for a convex domain containing the origin of $\mathbb{C}^{n}$, the gauge relative to 0 is defined by

$$
g(z)=\inf \{t \geq 0 \text { such that } z \in t \Omega\} .
$$

Then it is easy to see that $g$ is convex, smooth outside the origin and of finite type in a neighborhood of $\partial \Omega$. Thus the defining function we choose is smooth.

Note that, for the convex case, we are not able, in general, to get complete geometric separation of $\widetilde{\Omega}$ near $\partial \Omega \times\{0\}$ if we use a Diederich-Fornæss defining function (see Proposition 2.4). This property being indispensable in this section we need to use another defining function defined using the gauge: doing this, we loose the property of finite type everywhere on $\partial \widetilde{\Omega}$ and so the global properties of the Bergman projection $P^{\widetilde{\Omega}}$ but local estimates of the kernel of $P^{\widetilde{\Omega}}$ will suffice for our purpose.

\subsection{The case of finite type domains in $\mathbb{C}^{2}$}

\subsubsection{Precise comparison between the geometries of $\Omega$ and $\widetilde{\Omega}$}

We assume that the defining function $\rho$ of $\Omega$ is $\rho=\rho_{s}$ where $\rho_{s}$ satisfies (2.2) as stated in Section 2.1 and that the function $h$ satisfies Condition IV of Section 2.2 (so that $\widetilde{\Omega}$ is of finite type and has a Levi form locally diagonalizable at every point of $\partial \widetilde{\Omega}$ ).

We use the notations of the proof of Theorem 2.1 for $L, N, L_{1}$ and $W_{k}$, and let us denote by $\widetilde{N}$ the complex normal to the defining function $r$ of $\widetilde{\Omega}$ (i.e. $\widetilde{N}(r) \equiv 1$ in a neighborhood of the boundary of $\widetilde{\Omega}$ ). Moreover, for the geometries, using the notation " $\left.F^{\Omega}(L, z, \delta)\right)$ " introduced in Section 2 of [CD08], we denote $\widetilde{F}_{1}(\widetilde{\zeta}, \delta)=\delta^{-2}, \widetilde{F}_{2}(\widetilde{\zeta}, \delta)=F^{\widetilde{\Omega}}\left(L_{1}, \widetilde{\zeta}, \delta\right), \widetilde{F}_{i}(\widetilde{\zeta}, \delta)=$ $F^{\widetilde{\Omega}}\left(W_{i-2}, \widetilde{\zeta}, \delta\right)$ and $F_{L}(\zeta, \delta)=F^{\Omega}(L, \zeta, \delta)$.

Let us first compare the weights $\widetilde{F}_{i}(\widetilde{\zeta}, \delta)$ and $F_{L}(\zeta, \delta)$ constructed with the extremal basis defined in Section 2.5.

Lemma 4.1. We have:

(1) $L_{1}=L-\sum * W_{k}=L-\sum * \frac{\partial}{\partial w_{i}}-\left(\sum * \frac{\partial h_{i}}{\partial w_{i}}\right) N$, where $*$ are $\mathscr{C}^{\infty}$ functions;

(2) $N=\beta \widetilde{N}+\sum\left(* \frac{\partial h_{i}}{\partial \bar{w}_{i}}+\sum_{j>i} *\left|\frac{\partial h_{j}}{\partial w_{j}}\right|^{2}\right) W_{i}$, where $\beta$ and $*$ are $\mathscr{C}^{\infty}$ functions, $\beta \simeq 1$ for $|w|$ small.

Proof. Part (1) is a trivial consequence of the definitions of the vector fields. Let us give some indications for part (2). We have

$$
\widetilde{N}=\frac{\overline{\nabla \rho}+\overline{\nabla h}}{|\nabla \rho|^{2}+|\nabla h|^{2}} \text { and } N=\frac{\overline{\nabla \rho}}{|\nabla \rho|^{2}} .
$$

Thus

and

$$
N=\beta \widetilde{N}+* \sum \frac{\partial h_{i}}{\partial \overline{w_{i}}}\left(Z_{i}+\frac{\partial h_{i}}{\partial \overline{w_{i}}} N\right)
$$

$$
\left(1-\sum *\left|\frac{\partial h_{i}}{\partial w_{i}}\right|^{2}\right) N=\beta \widetilde{N}+\sum\left(* \frac{\partial h_{i}}{\partial \overline{w_{i}}}+\sum_{j>i} *\left|\frac{\partial h_{j}}{\partial w_{j}}\right|^{2}\right) W_{i}
$$

Now we apply this lemma to estimate the weights $\widetilde{F}_{i}(\widetilde{\zeta}, \delta)$ for $\widetilde{\zeta}=(\zeta, 0) \in \partial \widetilde{\Omega}$.

Denoting $c_{11}=[L, \bar{L}](\partial \rho)$, we have

$$
\begin{aligned}
\widetilde{c}_{11} & =\left[L_{1}, \overline{L_{1}}\right](\partial r) \\
& =\left[L-\sum * \frac{\partial}{\partial w_{i}}-\left(\sum * \frac{\partial h_{i}}{\partial \overline{w_{i}}}\right) N, \bar{L}-\sum * \frac{\partial}{\partial \overline{w_{i}}}-\left(\sum * \frac{\partial h_{i}}{\partial \overline{w_{i}}}\right) \bar{N}\right](\partial r) \\
& =c_{11}+\sum * \frac{\partial h_{i}}{\partial w_{i}}+\sum * \frac{\partial h_{i}}{\partial \overline{w_{i}}} .
\end{aligned}
$$

Then the order of the functions $h_{i}$ being greater than the type of $\Omega$, it is obvious that $\widetilde{F}_{1}(\widetilde{\zeta}, \delta) \simeq F_{1}(\zeta, \delta)$.

Furthermore, in the proof of Theorem 2.1, we saw that $\left[W_{k}, \overline{W_{k}}\right](\partial r)=\alpha_{k} \frac{\partial^{2} h_{k}}{\partial w_{k} \partial \overline{w_{k}}}, \alpha_{k} \in \mathscr{C}^{\infty},\left|\alpha_{k}\right| \geq 1 / 2$, then, as $W_{k}=\frac{\partial}{\partial w_{k}}+$ $* N+\sum_{i<k} * \frac{\partial}{\partial w_{i}}$ and

it follows clearly that

$$
\widetilde{F}_{k+2}=\sum_{\mathscr{L} \in \mathscr{L}_{k}}\left(\frac{\mathscr{L}\left(\left[W_{k}, \overline{W_{k}}\right](\partial r)\right)}{\delta}\right)^{2 /|\mathscr{L}|+2}
$$

$$
\widetilde{F}_{k+2}(\widetilde{\zeta}, \delta) \simeq\left(\frac{1}{\delta}\right)^{1 / q_{k}} \lesssim \widetilde{F}_{1}(\widetilde{\zeta}, \delta)
$$

Finally, we compare the pseudo-distances in $\Omega$ and $\widetilde{\Omega}$. 
Let $\gamma$ and $\widetilde{\gamma}$ be the respective pseudo-distances in $\partial \Omega$ and $\partial \widetilde{\Omega}$ defined by the exponential map of tangent vectors fields associated to extremal basis (see [CD08] and [CD06b, p. 75 and 100]). Then

Lemma 4.2. With the above notations, $\gamma(z, \zeta) \simeq \widetilde{\gamma}(\widetilde{z}, \widetilde{\zeta})$, for $z$ and $\zeta$ in $\partial \Omega$.

Proof. We use the notations introduced in [CD06b]: for $\Omega, \mathscr{Y} 1=\Re \mathrm{e} N, \mathscr{Y} / 2=\mathfrak{I m} N, \mathscr{Y}_{3}=\mathfrak{R e} L_{1}, \mathscr{Y}_{4}=\mathfrak{I} \mathrm{m} L_{1}$ and, for $\widetilde{\Omega}$, $\widetilde{\mathscr{Y}_{1}}=\Re \mathrm{e} \widetilde{N}, \widetilde{\mathscr{Y}} 2=\mathfrak{I m} \widetilde{N}, \widetilde{\mathscr{Y}}_{3}=\Re \mathrm{e} \widetilde{L}_{1}, \widetilde{\mathscr{Y}}_{4}=\mathfrak{I m} \widetilde{L}_{1}$ and $\widetilde{\mathscr{Y}}_{2 k+3}=\Re \mathrm{e} W_{k}, \widetilde{\mathscr{Y}}_{2 k+4}=\mathfrak{I m} W_{k}$. Then, following [CD06b, p. 100], there exists $\varphi:[0,1] \rightarrow \mathbb{C}^{2}$, piecewise $\mathscr{C}^{1}$, such that $\varphi(0)=z, \varphi(1)=\zeta, \varphi^{\prime}(t)=\sum a_{i}(t) \mathscr{Y}_{i}(\varphi(t))$ a.e. with $\left|a_{i}(t)\right| \lesssim G_{i}(z, \gamma(z, \zeta))^{-1 / 2}$, where $G_{1}=G_{2}=1 / \delta^{2}$ and $G_{3}=G_{4}=F_{1}(z, \delta)$. Now, let $\widetilde{\varphi}(t)=(\varphi(t), 0)$ be the same curve considered in $\mathbb{C}^{2+m}$. Then Lemma 4.1 and $\left.G_{1}(z, \delta)=\widetilde{G}_{1}(\widetilde{z}, \delta)=\frac{1}{\delta^{2}} \gtrsim F_{1}(z, \delta) \simeq \widetilde{F}_{1}(\widetilde{z}, \delta) \gtrsim \widetilde{F}_{k}(\widetilde{z}, \delta)=\widetilde{G}_{2 k+1}(\widetilde{z}, \delta)=\widetilde{G}_{2 k+2}(\widetilde{z}, \delta), k \geq 2\right)$ show that $\widetilde{\varphi}$ satisfies $\widetilde{\varphi}^{\prime}(t)=$ $\sum b_{i}(t) \widetilde{\mathscr{Y}_{i}}(\widetilde{\varphi}(t))$ with $\left|b_{i}(t)\right| \lesssim \widetilde{G}_{i}(\widetilde{z}, \gamma(z, \zeta))^{-1 / 2}$. Thus

$$
\widetilde{\gamma}(\widetilde{z}, \widetilde{\zeta}) \lesssim \gamma(z, \zeta)
$$

To show the converse inequality, consider a curve $\widetilde{\varphi}:[0,1] \rightarrow \mathbb{C}^{2+m}$ such that $\widetilde{\varphi}(0)=\widetilde{z}, \widetilde{\varphi}(1)=\widetilde{\zeta}, \widetilde{\varphi}^{\prime}(t)=\sum b_{i}(t) \widetilde{\mathscr{Y}}(\widetilde{\varphi}(t))$ a.e. with $\left|b_{i}(t)\right| \lesssim \widetilde{G}_{i}(\widetilde{z}, \widetilde{\gamma}(\widetilde{z}, \widetilde{\zeta}))^{-1 / 2}$.

First, we consider the component $\widetilde{\varphi}_{2+i}(t)$ of the curve $\widetilde{\varphi}$. Let us decompose $\widetilde{\varphi}^{\prime}$ on the basis $L, N$, $\frac{\partial}{\partial w_{i}}$ and their conjugates. Then the coefficient $\widetilde{w}_{i}\left(\widetilde{\varphi}^{\prime}(t)\right)$ of $\widetilde{\varphi}^{\prime}(t)$ in the directions $\frac{\partial}{\partial w_{i}}$ or $\frac{\partial}{\partial \bar{w}_{i}}$ is, in modulus, bounded from above by

$$
F_{1}(z, \widetilde{\gamma}(\widetilde{z}, \widetilde{\zeta}))^{-1 / 2}+\widetilde{F}_{i}(\widetilde{z}, \widetilde{\gamma}(\widetilde{z}, \widetilde{\zeta}))^{-1 / 2}+\widetilde{\gamma}(\widetilde{z}, \widetilde{\zeta}) \lesssim \widetilde{F}_{i}(\widetilde{z}, \widetilde{\gamma}(\widetilde{z}, \widetilde{\zeta}))^{-1 / 2} \lesssim \widetilde{\gamma}(\widetilde{z}, \widetilde{\zeta})^{1 / q_{i}}
$$

and thus, since $\widetilde{\varphi}_{2+i}(0)=0$,

$$
\left|\widetilde{\varphi}_{2+i}(t)\right| \lesssim \widetilde{\gamma}(\widetilde{z}, \widetilde{\zeta})^{1 / q_{i}}
$$

Now let us denote $\varphi(t)=\left(\widetilde{\varphi}_{1}(t), \widetilde{\varphi}_{2}(t)\right)$ the projection of $\widetilde{\varphi}$ onto $\mathbb{C}^{2}$, and let us write $\varphi^{\prime}(t)=\sum_{i=1}^{4} c_{i}(t) \mathscr{Y}(\varphi(t))$. We have to estimate the contribution of the coefficients $b_{j}$ to the coefficient $c_{i}$.

Suppose $j>4$. The contribution to $c_{3}$ and $c_{4}$ is null and to $c_{1}$ and $c_{2}$ is bounded by $\frac{\partial h}{\partial w_{j^{\prime}}} \widetilde{F}_{j^{\prime}+2}(\widetilde{z}, \widetilde{\gamma}(\widetilde{z}, \widetilde{\zeta}))^{-1 / 2}$ (with an evident correspondence $\left.j^{\prime} \longleftrightarrow j\right)$. As $\left|\frac{\partial h_{j^{\prime}}}{\partial w_{j^{\prime}}}\right| \lesssim\left|w_{j^{\prime}}\right|^{2 q_{j^{\prime}}-1} \lesssim \widetilde{\gamma}(\widetilde{z}, \widetilde{\zeta})^{\frac{2 q_{j^{\prime}}-1}{2 q_{j^{\prime}}}}$, this contribution is bounded by

$$
\widetilde{\gamma}(\widetilde{z}, \widetilde{\zeta})^{\frac{2 q_{j^{\prime}}-1}{2 q_{j^{\prime}}}} \widetilde{\gamma}(\widetilde{z}, \widetilde{\zeta})^{\frac{1}{2 q_{j^{\prime}}}}=\widetilde{\gamma}(\widetilde{z}, \widetilde{\zeta}) \text {. }
$$

If $j=4$ or 3 , the contribution to $c_{4}$ and $c_{3}$ is bounded by $\widetilde{\gamma}(\widetilde{z}, \widetilde{\zeta})$, and the contribution to $c_{1}$ and $c_{2}$ is bounded by

$$
\sum\left|\frac{\partial h_{i}}{\partial w_{i}}\right| \widetilde{F}_{1}(\widetilde{z}, \widetilde{\gamma}(\widetilde{z}, \widetilde{\zeta}))^{-1 / 2} \lesssim \widetilde{\gamma}(\widetilde{z}, \widetilde{\zeta})
$$

When $j=1$ or 2 , the contribution is bounded by $\widetilde{\gamma}(\widetilde{z}, \widetilde{\zeta})$.

This proves the lemma.

\subsubsection{Pointwise estimate of the Bergman kernel}

Theorem 4.1. Assume that $\Omega$ is pseudo-convex of finite type in $\mathbb{C}^{2}$ and that the hypotheses on $\rho$, $h$, stated at the beginning of the section are satisfied. Let $L$ be the complex tangent vector field to $\rho$ defined by $L=\frac{\partial \rho}{\partial z_{2}} \frac{\partial}{\partial z_{1}}-\frac{\partial \rho}{\partial z_{1}} \frac{\partial}{\partial z_{2}}$ and $N$ be the normal one such that $N \rho \equiv 1$ in a small neighborhood $U$ of $\partial \Omega$. Let $\mathscr{L}$ be a list of vector fields belonging to $\{L, \bar{L}, N, \bar{N}\}$.

Let us denote by $K_{\omega}^{\Omega}$ the Bergman kernel of $L_{\omega}^{2}(\Omega)$ for the weight $\omega$. Then for sufficiently close points $p_{1}$ and $p_{2}$ in $U$, we have the following estimate:

$$
\begin{aligned}
\left|\mathscr{L} K_{\omega}^{\Omega}\left(p_{1}, p_{2}\right)\right| & \leq C_{|\mathscr{L}|}\left(\frac{1}{\delta\left(p_{1}, p_{2}\right)^{2}}\right)^{1+l_{N} / 2} F_{L}^{1+l_{L} / 2}\left(p_{1}, \delta\left(p_{1}, p_{2}\right)\right) \prod_{j=1}^{m}\left(\frac{1}{\delta\left(p_{1}, p_{2}\right)}\right)^{1 / q_{j}} \\
& \simeq C_{|\mathscr{L}|} \frac{\left(\frac{1}{\delta\left(p_{1}, p_{2}\right)^{2}}\right)^{l_{N} / 2} F_{L}^{l_{L} / 2}\left(p_{1}, \delta\left(p_{1}, p_{2}\right)\right)}{\operatorname{Vol}_{\omega}\left(B\left(p_{1}, \delta\left(p_{1}, p_{2}\right)\right)\right)},
\end{aligned}
$$

where $F_{L}$ is the weight associated to $L, l_{L}$ (resp. $l_{N}$ ) denotes the number of times $L$ or $\bar{L}$ (resp. $N$ or $\bar{N}$ ) appears in the list $\mathscr{L}, \delta\left(p_{1}, p_{2}\right)=\left|\rho\left(p_{1}\right)\right|+\left|\rho\left(p_{2}\right)\right|+\delta_{\Omega}\left(p_{1}, p_{2}\right), \delta_{\Omega}\left(p_{1}, p_{2}\right)=\gamma\left(\pi\left(p_{1}\right), \pi\left(p_{2}\right)\right), \gamma$ being the pseudo-distance on $\partial \Omega$, and $B\left(p_{1}, \delta\left(p_{1}, p_{2}\right)\right)$ the associated pseudo-ball, of the geometry describe in [CD06b] and Vol $\omega$ denotes the volume with respect to the measure $\omega(z) d \lambda(z)$. 
Proof. We use (3) of Lemma 3.2 and the sharp estimates on $K_{B}^{\widetilde{\Omega}}$ deduced from [CD06b].

Suppose $|\mathscr{L}|=0$. We have $\left|K_{B}^{\widetilde{\Omega}}(\widetilde{z}, \widetilde{\zeta})\right| \lesssim \operatorname{Vol}(\widetilde{B}(\widetilde{z}, \widetilde{\delta}(\widetilde{z}, \widetilde{\zeta})))^{-1}$, by [CD06b, Main Theorem on the Bergman kernel, part II, p. 77], with, by Lemma 4.2,

$$
\widetilde{\delta}(\widetilde{z}, \widetilde{\zeta})=|r(\widetilde{z})|+|r(\widetilde{\zeta})|+\delta_{\widetilde{\Omega}}(\widetilde{z}, \widetilde{\zeta}) \simeq|\rho(z)|+|\rho(\zeta)|+\delta_{\Omega}(z, \zeta)=: \delta
$$

and, by [CD06b, Section 3], $\left|K_{B}^{\widetilde{\Omega}}(\widetilde{z}, \widetilde{\zeta})\right| \lesssim \operatorname{Vol}(\widetilde{B}(\widetilde{\pi(z)}, \widetilde{\delta}(\widetilde{z}, \widetilde{\zeta})))^{-1}$.

The estimates of the functions $F_{i}$ and $\widetilde{F}_{i}$ in the proofs of the lemmas show that

$$
\operatorname{Vol}(\widetilde{B}(\widetilde{\pi(z)}, \delta)) \simeq \delta^{2} \widetilde{F}_{1}(\widetilde{\pi(z)}, \delta) \prod_{k=1}^{m} \delta^{1 / q_{k}} \simeq \delta^{2+\sum_{k} 1 / q_{k}} F_{1}(z, \delta) .
$$

To finish the proof, we have to estimate $\operatorname{Vol}(B(z, \delta))=\int_{B(z, \delta)} \omega(\xi) d V(\xi)$ :

$$
\begin{aligned}
\omega(\xi) & =\operatorname{Vol}\{w \text { such that } h(w)<-\rho(\xi)\} \\
& \simeq \prod \operatorname{Vol}\left\{w_{i} \text { such that } h_{i}\left(w_{i}\right)<\rho(\xi)\right\} \simeq \prod(-\rho(\xi))^{\sum 1 / q_{i}} .
\end{aligned}
$$

Then, using the fact that $\xi \in B(z, \delta)$ and $|\rho(z)| \lesssim \delta$ imply $|\rho(\xi)| \lesssim \delta$ (see [CD06b, CD08]), we obtain

$$
\operatorname{Vol}(B(z, \delta)) \simeq \delta^{2+\sum 1 / q_{i}} F_{1}(z, \delta)^{-1}
$$

which finishes the proof when $\|\mathscr{L}\|=0$.

When $|\mathscr{L}| \geq 1$, the proof is done similarly using Lemmas 4.1 and 4.2 and the inequalities on the derivatives of $K_{B}^{\widetilde{\Omega}}$ given in [CD06b].

Remark. This proof easily generalizes in higher dimensions $n$ when the Levi form of $\rho$ has a rank $\geq n-2$.

\subsection{The case of convex domains of finite type in $\mathbb{C}^{n}$}

Now we assume that the function $h$ satisfies Condition V of Section 2.2 (for example $h(w)=\sum\left|w_{i}\right|^{2 q_{i}}, w_{i} \in \mathbb{C}$ ).

\subsubsection{Choice of the defining function and geometry of $\widetilde{\Omega}$}

Because of Remark 2.4, we have to choose a special defining function to obtain useful properties on $\widetilde{\Omega}$.

Let $g$ be the gauge function for $\Omega$. Then $\rho=g^{4} e^{1-1 / g}-1$ is a smooth convex defining function for $\Omega$ which is of finite type in a neighborhood of $\partial \Omega$. Thus the domain $\widetilde{\Omega}=\left\{(z, w) \in \mathbb{C}^{n} \times \mathbb{C}^{m}\right.$ such that $\left.\rho(z)+h(w)<0\right\}$ is smooth, convex and of finite type in a neighborhood of $\partial \Omega \times\{0\}$.

To get a useful estimate of the Bergman kernel of $\widetilde{\Omega}$, we need a precise comparison between the geometries of $\partial \Omega$ and of $\partial \widetilde{\Omega}$ near the points of $\partial \Omega \times\{0\}$.

Let $P_{0} \in \partial \Omega, \widetilde{P}_{0}=\left(P_{0}, 0\right) \in \partial \widetilde{\Omega}$ and $\delta>0$ sufficiently small. We now investigate extremal bases at $P_{0}$ and $\widetilde{P}_{0}$ (in the sense of [McN94, Hef04]).

Lemma 4.3. Let $\left(z_{1}^{\delta}, \ldots, z_{n}^{\delta}\right)$ be a $\delta$-extremal coordinate system at $P_{0}$. Then it is possible to choose a $\delta$-extremal coordinate system at $\widetilde{P}_{0},\left(\widetilde{z}_{1}^{\delta}, \ldots, \widetilde{z}_{m+n}^{\delta}\right)$, such that, for $1 \leq i \leq n, \widetilde{z}_{i}^{\delta}=\left(z_{i}^{\delta}, 0\right)$.

Proof. Let

$$
\widetilde{H_{\delta}}=\left\{\widetilde{P} \in \mathbb{C}^{n+m} \text { such that } r(\widetilde{P})=\delta\right\} .
$$

Let $\widetilde{P}_{1} \in \widetilde{H_{\delta}}$ such that $\left|\widetilde{P}_{1}-\widetilde{P}_{0}\right|$ is the euclidean distance $d\left(\widetilde{P_{0}}, \widetilde{H_{\delta}}\right)$ from $\widetilde{P}_{0}$ to $\widetilde{H_{\delta}}$. Let $Q_{1}$ be the projection of $\widetilde{P}_{1}$ to $\mathbb{C}^{n}$ so that $\widetilde{P}_{1}=\left(Q_{1}, w\right)$. We have

$$
\delta-\rho\left(Q_{1}\right)=h(w) \asymp \sum\left|w_{i}\right|^{2 q_{i}},
$$

and, by the condition on the $q_{i}$ (recall that $h$ satisfies Condition $\mathrm{V}$ and then $q_{i}>\operatorname{typ}(\Omega)=\tau$ ) we obtain

$$
|w|^{2} \gtrsim\left(\delta-\rho\left(Q_{1}\right)\right)^{2 / 2 \tau+2} .
$$

On the other hand, the geometric properties of $\Omega$ show that there exists

$$
Q_{2} \in H_{\delta}=\left\{P \in \mathbb{C}^{n} \text { such that } \rho(P)=\delta\right\}
$$

such that the distance $d\left(Q_{2}, H_{\delta}\right)$ from $Q_{2}$ to $H_{\delta}$ is less than $C\left(\delta-\rho\left(Q_{1}\right)\right)^{1 / \tau}$ (with a constant $C$ independent of $\delta$ ), and, by the definition of $\widetilde{P_{1}}$,

$$
\left|P_{1}-Q_{1}\right|^{2}+c\left(\delta-\rho\left(Q_{1}\right)\right)^{2 / 2 \tau+2} \leq\left(\left|P_{1}-Q_{1}\right|+C\left(\delta-\rho\left(Q_{1}\right)\right)^{1 / \tau}\right)^{2}
$$

which implies, for $\delta$ small enough (depending on $c, C$ and $\tau$, i.e. on $\Omega$ ), $\rho\left(Q_{1}\right)=\delta$ and we can choose $\widetilde{z}_{1}^{\delta}=\left(z_{1}^{\delta}, 0\right)$.

Define now $\widetilde{H}_{2, \delta}$ as the intersection of $\widetilde{H}_{\delta}$ with the affine complex space orthogonal to $\widetilde{z}_{1}^{\delta}$ passing through $\widetilde{P}_{0}$. Let $\widetilde{P}_{2}$ be such that $\left|\widetilde{P}_{2}-\widetilde{P}_{0}\right|$ is the euclidean distance from $\widetilde{P}_{0}$ to $\widetilde{H}_{2, \delta}$. Let $w_{0} \in \partial \Omega$ and $U$ be a small neighborhood of $w_{0}$. Arguing as before, it is easy to show that $\widetilde{P}_{2} \in \partial \Omega \times\{0\}$ and we can choose $\widetilde{z}_{2}^{\delta}=\left(z_{2}^{\delta}, 0\right)$. The proof is finished by induction. 
Corollary. Let $L_{1}, \ldots, L_{n}$ be the $\delta$-extremal basis of vector fields associated to the $\delta$-extremal coordinate system at the point $P_{0} \in \partial \Omega \cap U$ defined in Lemma 4.3 (see [CD08]). Then the basis $\widetilde{L}_{1}, \ldots, \widetilde{L}_{n+m}$ defined by:

(1) for $1 \leq i \leq n, L_{i}=\widetilde{L}_{i}$,

(2) for $1 \leq j \leq m, \widetilde{L}_{n+j}=\frac{\partial}{\partial w_{j}}-\beta_{n+j} \frac{\partial}{\partial Z_{1}}$, where $Z_{1}$ is the complex normal to $\partial \Omega$ at the point $w_{0}$, $\beta_{n+j}$ being so that $\widetilde{L}_{n+j}$ is tangent to $\partial \widetilde{\Omega}$,

is $\delta$-extremal at $\widetilde{P}_{0}$.

Proof. For the point (1), note that, for $i \geq 2, L_{i}=\frac{\partial}{\partial z_{i}}-\beta_{i} \frac{\partial}{\partial Z_{1}}$ (see [CD08, Section 7.1]) (recall that $\left.L_{1}=\widetilde{L}_{1}=N\right)$. For (2), without loss of generality, we can assume $q_{j+1} \geq q_{j}, 1 \leq j \leq m-1$, and the result is trivial if $h_{i}\left(w_{i}\right)=\left|w_{i}\right|^{2 q_{i}}$ and "easy" to prove in the general case using [CD08].

Let $F_{i}$ and $\widetilde{F}_{i}$ be the weights defined with the vector fields $L_{i}$ and $\widetilde{L}_{i}$. Then

Lemma 4.4. For $1 \leq i \leq n, F_{i}(z, \delta)=\widetilde{F}_{i}(\widetilde{z}, \delta)$ and, for $1 \leq j \leq m, \widetilde{F}_{n+j}(\widetilde{z}, \delta) \simeq\left(\frac{1}{\delta}\right)^{1 / q_{j}}$, for $z \in U$ and $\widetilde{z}=(z, 0)$.

Proof. The first part is a trivial consequence of the preceding corollary and the second is proved, as in the case of dimension 2 , noting that $\beta_{n+j}=-\frac{\partial h_{j}\left(w_{j}\right)}{\partial w_{j}} / \frac{\partial \rho}{\partial Z_{1}}$, with $\frac{\partial \rho}{\partial Z_{1}} \mathscr{C}^{\infty}$ and close to 1 for $\delta$ small.

\subsubsection{Pointwise estimate of the Bergman kernel}

Theorem 4.2. Assume $\Omega$ is convex of finite type in $\mathbb{C}^{n}$ and that the hypothesis on $\rho, h$, and $\omega$ stated at the beginning of the section are satisfied. Let $w_{0}$ be a boundary point of $\Omega$ and $U$ a small neighborhood of $w_{0}$. Let $N$ be the complex normal to $\partial \Omega$ (i.e. $N \rho \equiv 1$ in a neighborhood $U$ of $\partial \Omega$ ). Let $p_{1}$ and $p_{2}$ be two points in $U$ and $\delta_{\Omega}\left(p_{1}, p_{2}\right)$ as in Theorem 4.1 . Let $\left\{L_{2}, \ldots, L_{n}\right\}$ be a $\delta\left(p_{1}, p_{2}\right)$-extremal basis associated to $\rho$ at the point $p_{1}$ (with $\delta\left(p_{1}, p_{2}\right)=\left|\rho\left(p_{1}\right)\right|+\left|\rho\left(p_{2}\right)\right|+\delta_{\Omega}\left(p_{1}, p_{2}\right)$ ). Let us denote $L_{1}=N$. Let $\mathscr{L}$ be a list of vector fields belonging to $\left\{L_{1}, \overline{L_{1}}, \ldots, L_{n}, \overline{L_{n}}, N, \bar{N}\right\}$. Let $K_{\omega}^{\Omega}$ be the Bergman kernel of $L_{\omega}^{2}(\Omega)$ for the weight $\omega$. Then

$$
\begin{aligned}
\left|\mathscr{L} K_{\omega}^{\Omega}\left(p_{1}, p_{2}\right)\right| & \leq C_{|\mathscr{L}|}\left(\frac{1}{\delta\left(p_{1}, p_{2}\right)^{2}}\right)^{1+l_{N} / 2} \mathscr{F}^{1+\mathscr{L} / 2}\left(p_{1}, \delta\left(p_{1}, p_{2}\right)\right) \prod_{j=1}^{m}\left(\frac{1}{\delta\left(p_{1}, p_{2}\right)}\right)^{1 / q_{j}} \\
& \simeq C_{|\mathscr{L}|} \frac{\left(\frac{1}{\delta\left(p_{1}, p_{2}\right)^{2}}\right)^{l_{N} / 2} \mathscr{F}^{\mathscr{L} / 2}\left(p_{1}, \delta\left(p_{1}, p_{2}\right)\right)}{\operatorname{Vol}_{\omega}\left(B\left(p_{1}, \delta\left(p_{1}, p_{2}\right)\right)\right)},
\end{aligned}
$$

where $l_{N}$ denotes the number of times $N$ or $\bar{N}$ appears in the list $\mathscr{L}, \mathscr{F}^{1+\mathscr{L} / 2}\left(p_{1}, \delta\left(p_{1}, p_{2}\right)\right)=\prod_{i=2}^{n} \mathscr{F}_{L_{i}}^{1+l_{i} / 2}, l_{i}$ being the number of times $L_{i}$ or $\overline{L_{i}}$ appears in the list $\mathscr{L}$ and $V_{0} l_{\omega}$ denotes the volume with respect to the measure $\omega(z) d \lambda(z)$.

Proof. The construction made before shows that the estimate is immediate because, the exponential map being a local diffeomorphism ([CD06b, p. 75]), the fact that $L_{i}=\widetilde{L}_{i}, 1 \leq i \leq n$ (corollary of Lemma 4.3), implies $\delta_{\Omega}\left(p_{1}, p_{2}\right)=\delta_{\widetilde{\Omega}}\left(p_{1}, p_{2}\right)$.

\subsection{Proof of Theorem 1.1}

In the two cases we consider here, $\partial \widetilde{\Omega}$ is of finite type at every point of the form $(z, 0)$. Then, by Catlin's theorem ([Cat87]), the results of [KN65] show that the Neumann operator of $\widetilde{\Omega}$ is pseudolocal at these points, and, the method introduced by N. Kerzman in [Ker72] proves that the restriction of the Bergman kernel of $\widetilde{\Omega}$ to $(\bar{\Omega} \times\{0\})^{2}$ is $\mathscr{C}^{\infty}$ outside the diagonal of $(\partial \Omega \times\{0\})^{2}$. Thus, the identity $K_{\omega}^{\Omega}\left(p_{1}, p_{2}\right)=K^{\widetilde{\Omega}}\left(\left(p_{1}, 0\right),\left(p_{2}, 0\right)\right)$ implies that the estimates of Theorems 4.1 and 4.2 are valid everywhere.

These estimates, the hypothesis on $h$ (i.e. $\left.h(w) \asymp \sum\left|w_{i}\right|^{2 q_{i}}\right)$, an immediate generalization of Proposition 2.1 of [BCG96] and a standard application of Hölder inequality imply that $P_{\omega}^{\Omega}$ maps $L^{p}\left(\Omega,(-\rho)^{\alpha} d \lambda\right)$ continuously into itself for $-1<\alpha<$ $p\left(1+\sum \frac{1}{q_{i}}\right)-1$.

The Lipschitz estimate is also standard.

Now, choosing the special function $h(w)=\sum\left|w_{i}\right|^{2 q_{i}}, w_{i} \in \mathbb{C}$, the weight $\omega$ is equal to $C(-\rho)^{\sum 1 / q_{i}}$, and Theorem 1.1 follows. Remark. Note that same method gives trivially Theorem 1.1 for pseudo-convex decoupled domains of finite type in $\mathbb{C}^{n}$.

\section{REFERENCES}

[BC00] Bo Berndtsson and Philippe Charpentier, A Sobolev mapping property of the Bergman kernel, Math. Z. 235 (2000), no. 1, 1-10.

[BCG96] A. Bonami, D. Chang, and S. Grellier, Commutation properties and Lipschitz estimates for the Bergman and Szegö projections, Math. Z. 223 (1996), 275-302.

[BG95] A. Bonami and S. Grellier, Weighted Bergman projections in domains of finite type in $\mathbb{C}^{2}$, Contemp. Math. 189 (1995), 65-80.

[BS91] Harold P. Boas and Emil J. Straube, Sobolev estimates for the $\bar{\partial}$-Neumann operator on domains in $\mathbb{C}^{n}$ admitting a defining function that is plurisubharmonic on the boundary, Math. Z. 206 (1991), no. 1, 81-88.

[Cat87] David Catlin, Subelliptic estimates for the $\bar{\partial}$-Neumann problem on pseudoconvex domains, Ann. of Math. 126 (1987), no. 1, $131-191$.

[CD06a] P. Charpentier and Y. Dupain, Estimates for the Bergman and Szegö projections for pseudo-convex domains of finite type with locally diagonalizable Levi form, Publications Mathemàtiques 50 (2006), no. 2, 413-446. 
[CD06b] _ Geometry of Pseudo-convex Domains of Finite Type with Locally Diagonalizable Levi Form and Bergman Kernel, Jour. Math. Pures et Appl. 85 (2006), 71-118.

[CD08] _ Extremal Basis, Geometrically Separated Domains and Applications, http://fr.arxiv.org/abs/0810.1884 (2008).

[Cha80] P. Charpentier, Solutions minimales de l'équation $\bar{\partial} u=f$ dans la boule et le polydisque, Ann. Inst. Fourier 30 (1980), $121-153$.

[Cho96] S. Cho, Estimates of the Bergman kernel function on certain pseudoconvex domains in $\mathbb{C}^{n}$, Math Z. 222 (1996), 329-339.

[Cho03] B_ Boundary behavior of the Bergman kernel function on pseudoconvex domains with comparable Levi form, J. Math. Anal. Appl. 283 (2003), 386-397.

[Chr96] M. Christ, Global $\mathscr{C}^{\infty}$ irregularity of the $\bar{\partial}$-Neumann problem for worm domains, J. Amer. Math. Soc. 9 (1996), no. 4, $1171-1185$.

[CL97] Chang D. C. and Li B. Q., Sobolev and Lipschitz estimates for weighted Bergman projections, Nagoya Math. J. 147 (1997), 147-178.

[CNS92] D. C. Chang, A. Nagel, and E. Stein, Estimates for the $\bar{\partial}$-Neumann problem for pseudoconvex domains in $\mathbb{C}^{2}$ of finite type, Acta Math. 169 (1992), $153-228$.

[Cum90] A. Cumenge, Estimations limites pour la solution canonique de l'équation $\bar{\partial} u=f$, Math. Ann. 286 (1990), no. 4, $639-654$.

[DF77a] K. Diederich and J. E. Fornæss, Pseudoconvex Domains : Bounded Strictly Plurisubharmonic Exhaustion Functions, lnventiones math. 39 (1977), $129-141$.

[DF77b] _ Pseudoconvex domains: An example with nontrivial nebenhuelle, Math. Ann. 225 (1977), $275-292$.

[FR75] F. Forelli and W. Rudin, Projections on Spaces of Holomorphic Functions in Balls, Indiana Univ. Math. J. 24 (1975), no. 6, 593-602.

[Hef04] T. Hefer, Extremal bases and Hölder Estimates for $\bar{\partial}$ on Convex Domains of Finite Type, Michigan Math. J. 52 (2004), $573-602$.

[Hör65] L. Hörmander, $L^{2}$ estimates and existence theorems for the $\bar{\partial}$-operator, Acta Math. 113 (1965), 89-152.

[HP84a] R. Harvey and J. Polking, The $\bar{\partial}$-Neumann kernel in the ball in $\mathbb{C}^{n}$, Proc. Sympos. Pure Math. 41 (1984), $117-136$.

[HP84b] ㄴ The $\bar{\partial}$-Neumann solution to the inhomogeneous Cauchy-Riemann equation in the ball in $\mathbb{C}^{n}$, Trans. Amer. Math. Soc. 281 (1984), no. 2, $587-613$

[Ker72] N. Kerzman, The Bergman kernel function. Differentiability at the boundary, Math. Ann. 195 (1972), 149-158.

[KN65] J. J. Kohn and L. Nirenberg, Non coercive boundary value problems, Comm. Pure Appl. Math. 18 (1965), $443-492$.

[Koh73] J. J. Kohn, Global regularity for $\bar{\partial}$ on weakly pseudo-convex manifolds, Trans. Amer. Math. Soc. 181 (1973), $273-292$.

[Lig89] E. Ligocka, On the Forelli-Rudin construction and weighted Bergman projections, Studia Math. 94 (1989), no. 3, 257-272.

[LR86] I. Lieb and M. Range, Integral representations and estimates in the theory of the $\bar{\partial}$-Neumann problem., Ann. of Math. 123 (1986), no. 2, 265-301.

[LR87] ㄴ. The kernel of the $\bar{\partial}$-Neumann operator on strictly pseudoconvex domains, Math. Ann. 278 (1987), no. 1-4, $151-173$.

[LR88] _ The $\bar{\partial}$-Neumann kernel for codimension-one forms on strictly pseudoconvex domains, Math. Notes, Princeton Univ. Press 38 (1988), 473-782.

[McN94] J. McNeal, Estimates on Bergman Kernels of Convex Domains, Advances in Math (1994), 108-139.

[MS94] J. D. McNeal and E. M. Stein, Mapping properties of the Bergman projection on convex domains of finite type, Duke Math. J. 73 (1994), no. 1, $177-199$.

[MS97] _ The Szegö projection on convex domains, Math. Z. 224 (1997), no. 4, 519-553.

[NRSW89] A. Nagel, J. P. Rosay, E. M. Stein, and S. Wainger, Estimates for the Bergman and Szegö kernels in $\mathbb{C}^{2}$, Annals of Math. 129 (1989), $113-147$.

[PW90] Z. Pasternak-Winiarski, On the Dependance of the Reproducing Kernel on the Wieght of Integration, Jour. Func. Anal. 94 (1990), 110-134.

[Rud80] W. Rudin, Function theory in the unit ball of $\mathbb{C}^{n}$, Springer Verlag, 2008. - (Grundlehren der Mathematischen Wissenschaften; 241 ), 1980.

[Str10] E. Straube, Lectures on the $L^{2}$ Sobolev theory on the -Neumann problem, European Mathematical Society, $2010,2010$.

[Zeya] Y. Zeytuncu, $L^{p}$ regularity of some weighted Bergman projections on the unit disc, Turk. J. Math. To appear.

[Zeyb] $\quad L^{p}$ Regularity of weighted Bergman Projections, Tran. of Amer. Math. Soc. To appear.

[Zey11a] Sobolev regularity of weighted Bergman projections on the unit disc, Complex Variables and Elliptic Equations (2011).

[Zey11b] Weighted Bergman projections and kernels: $L^{p}$ regularity and zeros, Proc. Amer. Math. Soc. 139 (2011), $2105-2112$.

P. Charpentier \& Y. Dupain, Université Bordeaux I, Institut de Mathématiques de BordeauX, 351 , Cours de La Libération, 33405 , TALENCE, FRANCE

M. Mounkaila, Université Abdou Moumouni, FaCulté des Sciences, B.P. 10662, Niamey, Niger

E-mail address: P. Charpentier: philippe.charpentier@math.u-bordeaux1.fr

E-mail address: Y. Dupain: yves.dupain@math.u-bordeaux1.fr

E-mail address: M. Mounkaila: modi.mounkaila@yahoo.fr 